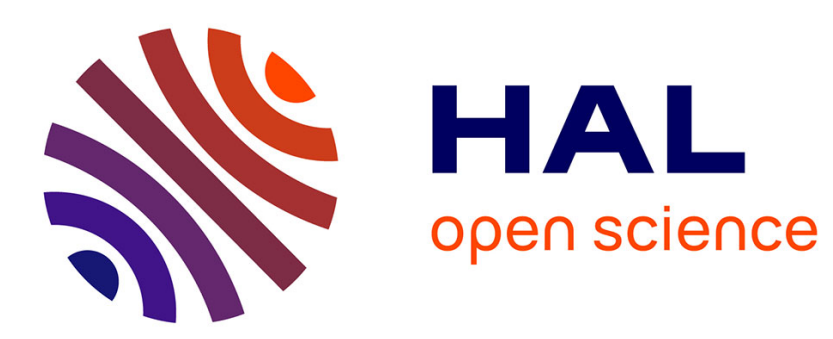

\title{
A Local Entropy Minimum Principle for Deriving Entropy Preserving Schemes
}

Christophe Berthon, Bruno Dubroca $\dagger \ddagger$, Afeintou Sangam

\section{To cite this version:}

Christophe Berthon, Bruno Dubroca $\dagger \ddagger$, Afeintou Sangam. A Local Entropy Minimum Principle for Deriving Entropy Preserving Schemes. SIAM Journal on Numerical Analysis, 2012, 10.1137/100814445. hal-01280691

\section{HAL Id: hal-01280691 \\ https://hal.inria.fr/hal-01280691}

Submitted on 29 Feb 2016

HAL is a multi-disciplinary open access archive for the deposit and dissemination of scientific research documents, whether they are published or not. The documents may come from teaching and research institutions in France or abroad, or from public or private research centers.
L'archive ouverte pluridisciplinaire HAL, est destinée au dépôt et à la diffusion de documents scientifiques de niveau recherche, publiés ou non, émanant des établissements d'enseignement et de recherche français ou étrangers, des laboratoires publics ou privés. 


\title{
A LOCAL ENTROPY MINIMUM PRINCIPLE FOR DERIVING ENTROPY PRESERVING SCHEMES.
}

\author{
CHRISTOPHE BERTHON *, BRUNO DUBROCA †‡, AND AFEINTOU SANGAM §
}

AMS subject classifications. 65M60, 65M12

Key words. Hyperbolic system, general Euler equations, Godunov type schemes, discrete entropy inequalities, discrete entropy minimum principle.

\begin{abstract}
The present work deals with the establishment of stability conditions of finite volume methods to approximate weak solutions of the general Euler equations to simulate compressible flows. In oder to ensure discrete entropy inequalities, we derive a new technique based on a local minimum principle to be satisfied by the specific entropy. Sufficient conditions are exhibited to satisfy the required local minimum entropy principle. Arguing these conditions, a class of entropy preserving schemes is thus derived.
\end{abstract}

1. Introduction. The numerical approximation of the weak solutions of hyperbolic systems of conservation laws was widely studied during the last three decades, with a special attention to the so-called Euler equations. Several strategies, coming from finite volume methods, have been introduced. Our purpose is not to detail these techniques, but let us refer to the most famous of them: the Godunov scheme [18, 25], the HLL scheme [21], the HLLC scheme [31], the Roe scheme [27], the Osher scheme [13], the relaxation schemes $[7,22,12,3,1]$, the VFRoe scheme $[9,5,17,26]$, BGK scheme $[18,23,8] \ldots$ Of course, this list stays exhaustive and the reader is referred, for instance, to Godlewski-Raviart [18] or Toro [32] or LeVeque [25] and references therein. These kind of schemes have also been applied to general fluid equations, for instance to 10-moment equations system [3, 28] or radiative transfer equations [4].

From the derivation of finite volume methods, the main questions arising concern the robustness, the stability and the accuracy of the suggested method. In the present work, we do not consider the delicate problem of the accuracy of the methods (see $[23,24,33,2]$ where several strategies are devised). Concerning the robustness, we classically admits this property as soon as the space of admissible states stays invariant by the numerical method. Most of the usual schemes (Godunov, HLL, HLLC...) are easily shown to be robust (see also $[14,15,16]$ ). However, some numerical approximations need a special attention to prove the required robustness (for instance, see [5] for an analysis of the VFRoe scheme). Now, some schemes violate this property (the initial Roe scheme for instance). When considering the stability statements, one adopts a discrete Lax entropy inequalities $[21,23,7]$. In general, the establishment of such discrete entropy inequalities are very difficult to be obtained. Except for the Godunov scheme or the HLL scheme where the proof is obvious [21], we need very sophisticated arguments to demonstrate an entropy preserving property (see [7, 1, 10] in the case of HLLC scheme and/or the Suliciu relaxation scheme).

\footnotetext{
*Laboratoire de Mathématiques Jean Leray, CNRS UMR 6629, Université de Nantes, 2 rue de la Houssinière, BP 92208, 44322 Nantes, France.

†Institut de Mathématiques de Bordeaux, CNRS UMR 5251, Université Bordeaux 1, 351, Cours de la Libération, 33405 Talence Cedex, France.

${ }^{\ddagger}$ Centre Lasers Intenses et Applications, CNRS UMR 5107, Université Bordeaux 1, 351, Cours de la Libération, 33405 Talence Cedex, France.

$\S$ Laboratoire J.-A. Dieudonné, CNRS UMR 6621, Université de Nice Sophia-Antipolis, Parc Valrose 06108 Nice, France.

๑INRIA Sophia-Antipolis 2004, Route des Lucioles, BP 93, 06902 Sophia-Antipolis Cedex, France.
} 
When focusing on the General Euler equations, we propose new arguments to ensure robustness and entropy preservation to be satisfied by the scheme. The interest of our approach is twofold. First, it yields an immediate establishment of the robustness and the satisfaction of discrete entropy inequalities of the HLLC scheme and relaxations schemes (for instance). From now on, let us note that we revisit these schemes and the reader is referred to $[7,1,10,6,14,15,16]$ where robustness and stability are given for the proposed schemes. The second interest of our approach comes from a derivation of a full class of robust and entropy preserving schemes to approximate the weak solutions of the General Euler equations. We will give an example which was, up to our knowledge, never proposed in the literature.

The present paper is devoted to the numerical approximation of the weak solutions of the general Euler equations. Hence we consider the following system:

$$
\left\{\begin{array}{l}
\partial_{t} \rho+\partial_{x} \rho u=0 \\
\partial_{t} \rho u+\partial_{x}\left(\rho u^{2}+p(\tau, e)\right)=0 \\
\partial_{t} \rho E+\partial_{x}(\rho E+p(\tau, e)) u=0
\end{array}\right.
$$

where $\rho>0$ is the density, $u \in \mathbb{R}$ the velocity and $\rho E>0$ the total energy. Here we have set $\tau$ the specific volume and $e$ the internal energy defined as follows:

$$
\tau=\frac{1}{\rho} \quad \text { and } \quad e=E-\frac{u^{2}}{2} .
$$

Concerning the pressure function $p$, we assume that it obeys the second law of thermodynamics. As a consequence, it exists a specific entropy $s(\tau, e): \mathbb{R}^{+} \times \mathbb{R}^{+} \rightarrow \mathbb{R}$ which satisfies for some temperature $T(\tau, e)>0$ :

$$
T d s=d e+p d \tau
$$

so that we have

$$
\frac{\partial s}{\partial \tau}(\tau, e)=\frac{p(\tau, e)}{T(\tau, e)}>0 \quad \text { and } \quad \frac{\partial s}{\partial e}(\tau, e)=\frac{1}{T(\tau, e)}>0 .
$$

In addition, we assume that the function $(\tau, e) \mapsto s(\tau, e)$ is strictly convex and it is asked, without restriction, to meet the following asymptotic condition for any given fixed $\tau>0$ :

$$
\lim _{e \rightarrow 0^{+}} s(\tau, e)=-\infty \text { and } \lim _{e \rightarrow+\infty} s(\tau, e)=+\infty
$$

Now, let us recall that the classical solutions of (1.1) satisfy the following additional transport law (see $[18,29])$ :

$$
\partial_{t} s(\tau, e)+u \partial_{x} s(\tau, e)=0 .
$$

It follows that any function $f$ of $s$ satisfies the same transport equation:

$$
\partial_{t} f(s(\tau, e))+u \partial_{x} f(s(\tau, e))=0 .
$$

Combining the continuity equation in (1.1) with (1.7) gives that smooth solutions of Euler equations (1.1) satisfy in addition a formal conservation law in the form:

$$
\partial_{t} \rho f(s(\tau, e))+\partial_{x} \rho f(s(\tau, e)) u=0 .
$$


This leads to a convex generalized entropy function $(\rho, \rho u, \rho E) \mapsto \rho f(s(\tau, e))$ such that the weak solutions of (1.1) satisfy the following entropy inequality (in the sense of distributions):

$$
\partial_{t} \rho f(s(\tau, e))+\partial_{x} \rho f(s(\tau, e)) u \leq 0 .
$$

From a numerical point of view this inequality will turn out to be crucial since it rules out some non-physical solutions. After the work by Harten et al [21, 19], the conditions to be satisfied in order to enforce the strict convexity of $(\rho, \rho u, \rho E) \mapsto \rho f(s(\tau, e))$ imply:

$$
f^{\prime}(s(\tau, e))<0 \quad \text { and } \quad p(\tau, e) \frac{\partial p}{\partial e}(\tau, e)-\frac{\partial p}{\partial \tau}(\tau, e)>0 .
$$

From now on, let us emphasize that these assumed conditions to be put on the pressure enforce the system (1.1) to be hyperbolic with eigenvalues $u-c, u$ and $u+c$ where the sound speed $c>0$ is given by

$$
c^{2}=\frac{1}{\rho^{2}}\left(p(\tau, e) \frac{\partial p}{\partial e}(\tau, e)-\frac{\partial p}{\partial \tau}(\tau, e)\right) .
$$

Let us add a supplementary property satisfied by the specific entropy. Indeed, after $[29,30,20], s(\tau, e)$ satisfies the following minimum principle:

$$
\inf _{x \in \mathbb{R}} s(\tau, e)(x, t) \leq \inf _{x \in \mathbb{R}} s(\tau, e)(x, 0) .
$$

This property will be, once again, relevant from a numerical point of view. We will ask the numerical approximations to satisfy such a minimum principle.

For the sake of simplicity, it is convenient to introduce notations to write (1.1) as follows:

$$
\partial_{t} U+\partial_{x} F(U)=0
$$

where the state vector $U$ and the vector flux function are given by:

$$
U=(\rho, \rho u, \rho E)^{T} \quad \text { and } \quad F(U)=\left(\rho u, \rho u^{2}+p(\tau, e),(\rho E+p(\tau, e)) u\right)^{T} .
$$

Here, the state vector $U$ belongs to an admissible state space $\Omega$ defined as follows:

$$
\Omega=\left\{U \in \mathbb{R}^{3} ; \rho>0, u \in \mathbb{R}, e>0\right\} .
$$

In the sequel, it will be helpful to introduce the following (nonstandard) state space:

$$
\omega=\left\{U \in \mathbb{R}^{3} ; \rho>0, u \in \mathbb{R}, E>0\right\} .
$$

In fact, we have $\Omega \subsetneq \omega$ and $\omega$ can not be considered to define admissible solutions with positive internal energy. However, this space $\omega$ will be useful to introduce some intermediate robustness properties.

The aim of the present paper concerns the derivation of approximate Riemann solvers to develop first-order finite volume schemes to approximate the weak solutions of (1.1). The derived schemes must be $\Omega$-invariant and preserve discrete entropy inequalities issuing from (1.8). The paper is organized as follows. In the next section, we introduce the main notations to define approximate Riemann solvers and 


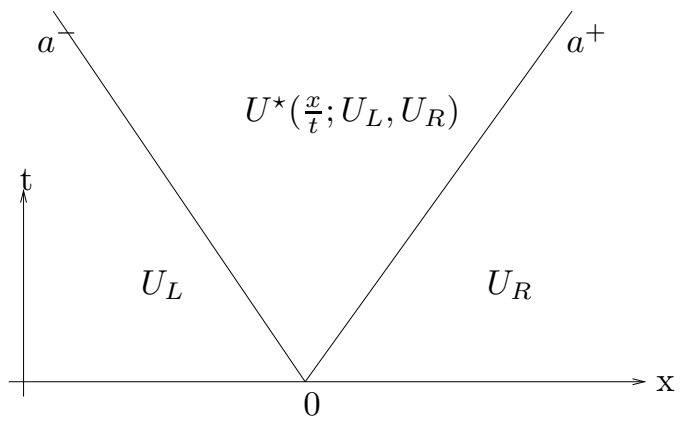

FIG. 2.1. Wave structure of the approximate Riemann solver

their associated Godunov type method. We recall the definition of robust schemes, entropy preserving schemes and entropy minimum principle preserving schemes. In addition, we complete this review by giving some basic results to enforce the required robustness and stability. In section 3 , we establish new criterion to obtain a new class of entropic schemes. In fact, after [23], the suggested criterion are based on a local entropy minimum principle to derive robust, entropy preserving and entropy minimum principle preserving schemes. The following section is devoted to apply the above results. In particular, we exhibit the stability properties satisfied by the Suliciu relaxation scheme $[7,12,1]$ or equivalently the HLLC scheme $[32,31]$. In the present work, the stability requirement needed by these two schemes will be seen as an easy corollary of our main result. In addition, we supplement this apply when deriving new entropy schemes. In the last section, we give a conclusion and we propose several extensions.

2. Godunov-type schemes. We here recall the main elements to derive a conservative first-order finite volume scheme of Godunov-type. Motivated by the work of Harten-Lax-van Leer [21], we introduce an approximation, denoted $U_{\mathcal{R}}^{\Delta x}\left(\frac{x}{t} ; U_{L}, U_{R}\right)$, of the exact Riemann solution we defined as follows (see Figure 2.1):

$$
U_{\mathcal{R}}^{\Delta x}\left(\frac{x}{t} ; U_{L}, U_{R}\right)=\left\{\begin{array}{l}
U_{L}, \quad \text { if } \frac{x}{t}<a^{-}, \\
U^{\star}\left(\frac{x}{t} ; U_{L}, U_{R}\right), \quad \text { if } a^{-}<\frac{x}{t}<a^{+}, \\
U_{R}, \quad \text { if } \frac{x}{t}>a^{+},
\end{array}\right.
$$

where $a^{-}<a^{+}$are some given constants we will define later on. The internal structure $U^{\star}\left(\frac{x}{t} ; U_{L}, U_{R}\right)$ can be simpler than the exact internal Riemann structure as long as it does not violate the conservation and the entropy inequalities.

Now, we consider this approximate Riemann solution $U_{\mathcal{R}}^{\Delta x}\left(\frac{x}{t} ; U_{L}, U_{R}\right)$ to define a finite volume scheme. We adopt a uniform structured mesh in space, defined by the cells $I_{i}=\left[x_{i-\frac{1}{2}}, x_{i+\frac{1}{2}}\right)$, where $x_{i+\frac{1}{2}}=x_{i}+\frac{\Delta x}{2}$ with a constant space increment $\Delta x$.

At the initial time, we set

$$
U_{i}^{0}=\frac{1}{\Delta x} \int_{x_{i-\frac{1}{2}}}^{x_{i+\frac{1}{2}}} U(x, 0) d x, \quad i \in \mathbb{Z} .
$$

At time $t^{n}$ we assume known a piecewise constant approximate solution $U^{\Delta x}\left(x, t^{n}\right)$ 


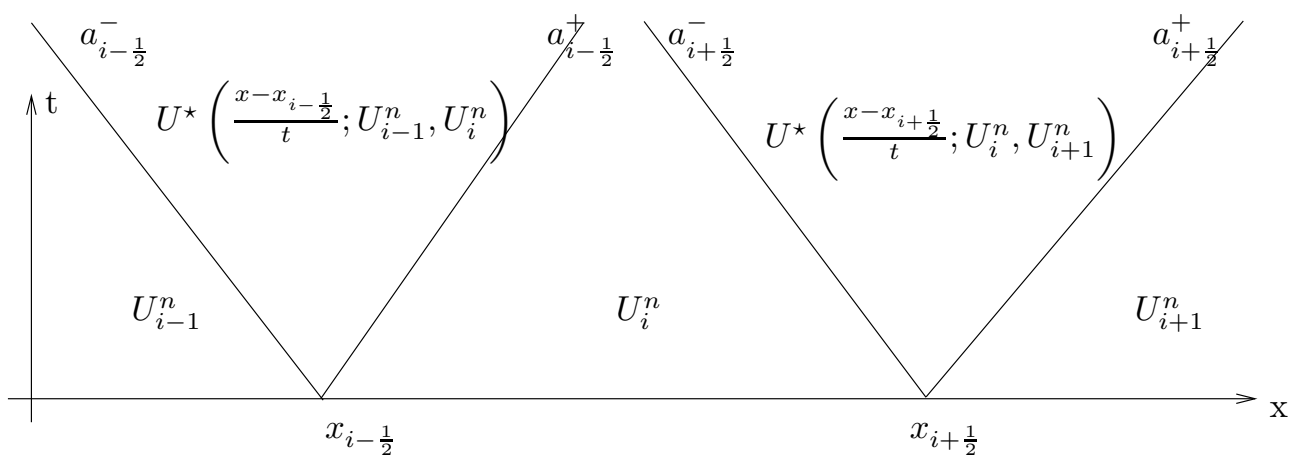

FIG. 2.2. Sequence of non interacting approximated Riemann solution

in $\Omega$ defined by

$$
U^{\Delta x}\left(x, t^{n}\right)=U_{i}^{n}, \quad x \in I_{i}, i \in \mathbb{Z} .
$$

This approximation is now evolved in time to define an approximate solution at time $t^{n+1}=t^{n}+\Delta t$. To address such an issue, at each cell interface, located at $x_{i+\frac{1}{2}}$, we consider the approximate Riemann solver $U_{\mathcal{R}}^{\Delta x}\left(\frac{x-x_{i+\frac{1}{2}}}{t} ; U_{i}^{n}, U_{i+1}^{n}\right)$. Under the CFL like restriction:

$$
\frac{\Delta t}{\Delta x} \max _{i \in \mathbb{Z}}\left(\left|a_{i+\frac{1}{2}}^{ \pm}\right|\right) \leq \frac{1}{2}
$$

we have thus defined a juxtaposition of non interacting approximate Riemann solutions for all $t \in[0, \Delta t$ ) (see Figure 2.2):

$$
U^{\Delta x}\left(x, t^{n}+t\right)=U_{\mathcal{R}}^{\Delta x}\left(\frac{x-x_{i+\frac{1}{2}}}{t} ; U_{i}^{n}, U_{i+1}^{n}\right), \quad x \in\left(x_{i}, x_{i+1}\right) .
$$

The updated approximate solution at time $t^{n+1}$ is then defined as follows:

$$
U_{i}^{n+1}=\frac{1}{\Delta x} \int_{x_{i-\frac{1}{2}}}^{x_{i+\frac{1}{2}}} U^{\Delta x}\left(x, t^{n}+\Delta t\right) d x .
$$

Due to Harten-Lax [19], the following statement shows that the adopted scheme is consistent:

THEOREM 2.1. Let $U_{\mathcal{R}}^{\Delta x}$ be an approximation of the Riemann solution that satisfies the following consistency condition:

$$
\frac{1}{\Delta x} \int_{-\frac{\Delta x}{2}}^{\frac{\Delta x}{2}} U_{\mathcal{R}}^{\Delta x}\left(\frac{x}{\Delta t} ; U_{L}, U_{R}\right) d x=\frac{1}{2}\left(U_{L}+U_{R}\right)-\frac{\Delta t}{\Delta x}\left(F\left(U_{R}\right)-F\left(U_{L}\right)\right),
$$

where $\Delta t$ is given by the CFL condition (2.2). Then the updated approximation (2.3) rewrites in conservation form as follows:

$$
U_{i}^{n+1}=U_{i}^{n}-\frac{\Delta t}{\Delta x}\left(F^{\Delta x}\left(U_{i}^{n}, U_{i+1}^{n}\right)-F^{\Delta x}\left(U_{i-1}^{n}, U_{i}^{n}\right)\right),
$$

where the numerical flux function is defined by

$$
F^{\Delta x}\left(U_{L}, U_{R}\right)=F\left(U_{L}\right)+\frac{\Delta x}{2 \Delta t} U_{L}-\frac{1}{\Delta t} \int_{-\frac{\Delta x}{2}}^{0} U_{\mathcal{R}}^{\Delta x}\left(\frac{x}{\Delta t} ; U_{L}, U_{R}\right) d x .
$$


Now, Let us introduce definitions to characterize the robustness and the stability of schemes. Concerning the robustness, we have:

DEFINITION 2.2. The scheme (2.5)-(2.6) is said positively invariant iff, for all $i$ in $\mathbb{Z}, U_{i}^{n+1} \in \Omega$ as soon as $U_{i}^{n} \in \Omega$.

The scheme is said weakly robust iff, for all $i$ in $\mathbb{Z}, U_{i}^{n} \in \omega$ implies $U_{i}^{n+1} \in \Omega$.

Next, we define an entropy preserving scheme:

DEFINITION 2.3. The scheme (2.5)-(2.6) is said entropy preserving iff, for all $i \in \mathbb{Z}$ the updated state vector $U_{i}^{n+1}$ satisfies:

$$
\frac{1}{\Delta t}\left(S_{i}^{n+1}-S_{i}^{n}\right)+\frac{1}{\Delta x}\left(\eta^{\Delta x}\left(U_{i}^{n}, U_{i+1}^{n}\right)-\eta^{\Delta x}\left(U_{i+1}^{n}, U_{i}^{n}\right)\right) \leq 0
$$

where $S_{i}^{n}=\rho_{i}^{n} f\left(s\left(\tau_{i}^{n}, e_{i}^{n}\right)\right)$ and the numerical entropy flux function satisfies the consistency condition $\eta^{\Delta x}(U, U)=\rho f(s(\tau, e)) u$.

We conclude by defining a discrete entropy minimum principle:

DEFINITION 2.4. The scheme (2.5)-(2.6) is said entropy minimum principle preserving iff $U_{i}^{n+1}$ satisfies for all $i$ in $\mathbb{Z}$ :

$$
s\left(\tau_{i}^{n+1}, e_{i}^{n+1}\right) \geq \min \left(s\left(\tau_{i-1}^{n}, e_{i-1}^{n}\right), s\left(\tau_{i}^{n}, e_{i}^{n}\right), s\left(\tau_{i+1}^{n}, e_{i+1}^{n}\right)\right) .
$$

Based on such definitions, we examine conditions to be put on the approximate Riemann solver (2.1) to obtain the required robustness and stability of the numerical scheme. According to the $L^{2}$-projection (2.3) to define the updated state, we immediately note that such an approach is weakly robust as soon as the approximate Riemann solver, $U_{\mathcal{R}}^{\Delta x}$ defined by (2.1), stays in $\omega$ for all $U_{L}$ and $U_{R}$ in $\omega$. This remark can be completed with the result stated by Harten-Lax-van Leer [21] concerning the discrete entropy inequalities:

THEOREM 2.5. Let $U_{\mathcal{R}}^{\Delta x}$ be an approximation of the Riemann solution which satisfies the consistency condition (2.4). Under the CFL condition (2.2), assume the following entropy consistency condition:

$$
\begin{aligned}
\frac{1}{\Delta x} \int_{-\frac{\Delta x}{2}}^{\frac{\Delta x}{2}} S\left(U_{\mathcal{R}}^{\Delta x}\left(\frac{x}{\Delta t} ; U_{L}, U_{R}\right)\right) d x \leq \\
\frac{1}{2}\left(S\left(U_{L}\right)+S\left(U_{R}\right)\right)-\frac{\Delta t}{\Delta x}\left(S\left(U_{R}\right) u_{R}-S\left(U_{L}\right) u_{L}\right),
\end{aligned}
$$

where we have set $S(U)=\rho f(s(\tau, e))$ for any given smooth function $f: \mathbb{R} \rightarrow \mathbb{R}$ so that the function $U \mapsto S(U)$ is strictly convex. Then the scheme (2.5)-(2.6) is entropy preserving.

We skip the proof of this well-known result (for instance, see [19, 21]).

From now on, let us note that this entropy preserving criterion (2.8) is certainly one of the most general condition we can find in the literature. As stated in [21], such condition can be easily applied to the one intermediate constant state $\left(U^{\star}\left(\frac{x}{t} ; U_{L}, U_{R}\right)=\right.$ cste in $\left.(2.1)\right)$ as suggested to derive the so-called HLL scheme. Now, this criterion seems too weak to be easily applied when considering more sophisticated schemes. For instance, we refer to $[7,6,10,1,3]$ where distinct arguments are proposed to establish the entropy preserving property for the HLLC scheme or the Suliciu relaxation method. Here, the main idea consists in introducing a stronger entropy preserving condition in order to easily ensure the stability requirements when 
considering the HLLC or relaxation schemes. In addition, we will see that this new condition will give a full class of entropy preserving and entropy minimum principle preserving schemes.

3. A local entropy minimum principle. Arguing the work by KhobalattePerthame [23] (see also [10] for relating ideas), our main assumption consists in enforcing a local entropy minimum principle into the approximate Riemann solver.

THEOREM 3.1. Let $U_{\mathcal{R}}^{\Delta x}$ be an approximate Riemann solver, defined by (2.1), satisfying the consistency condition (2.4) and so that $U_{\mathcal{R}}^{\Delta x}\left(\frac{x}{t} ; U_{L}, U_{R}\right) \in \Omega$ as long as $U_{L}$ and $U_{R}$ are in $\Omega$. Assume the existence of $a^{\star} \in\left(a^{-}, a^{+}\right)$to get the following decomposition of the intermediate state:

$$
U^{\star}\left(\frac{x}{t} ; U_{L}, U_{R}\right)= \begin{cases}U_{L}^{\star}\left(\frac{x}{t} ; U_{L}, U_{R}\right) & \text { if } \frac{x}{t} \in\left(a^{-}, a^{\star}\right), \\ U_{R}^{\star}\left(\frac{x}{t} ; U_{L}, U_{R}\right) & \text { if } \frac{x}{t} \in\left(a^{\star}, a^{+}\right) .\end{cases}
$$

Assume this decomposition satisfies an additional half consistency condition given by

$$
\frac{1}{\Delta x} \int_{a^{\star} \Delta t}^{\frac{\Delta x}{2}} \rho_{\mathcal{R}}^{\Delta x}\left(\frac{x}{\Delta t} ; U_{L}, U_{R}\right) d x=\frac{\rho_{R}}{2}-\frac{\Delta t}{\Delta x} \rho_{R} u_{R}
$$

If the following local entropy minimum principle:

$$
\begin{array}{ll}
s\left(\tau_{L}^{\star}\left(\frac{x}{t} ; U_{L}, U_{R}\right), e_{L}^{\star}\left(\frac{x}{t} ; U_{L}, U_{R}\right)\right) \geq s\left(\tau_{L}, e_{L}\right) & \text { if } \frac{x}{t} \in\left(a^{-}, a^{\star}\right), \\
s\left(\tau_{R}^{\star}\left(\frac{x}{t} ; U_{L}, U_{R}\right), e_{R}^{\star}\left(\frac{x}{t} ; U_{L}, U_{R}\right)\right) \geq s\left(\tau_{R}, e_{R}\right) & \text { if } \frac{x}{t} \in\left(a^{\star}, a^{+}\right),
\end{array}
$$

is satisfied, then the scheme (2.5)-(2.6) is positively invariant, entropy preserving and entropy minimum principle preserving under the CFL restriction (2.2).

Let us note from now on that the half consistency condition (3.2) (firstly given in $[14,15,16]$ in a more restrictive form), when withdrawn from the consistency condition (2.4), leads to an equivalent half consistency condition stated on the interval $\left(a^{-}, a^{\star}\right)$ as follows:

$$
\frac{1}{\Delta x} \int_{-\frac{\Delta x}{2}}^{a^{\star} \Delta t} \rho_{\mathcal{R}}^{\Delta x}\left(\frac{x}{\Delta t} ; U_{L}, U_{R}\right) d x=\frac{\rho_{L}}{2}+\frac{\Delta t}{\Delta x} \rho_{L} u_{L} .
$$

As detailed in Section 4, the HLLC scheme or the Siluciu relaxation scheme involve explicitely the intermediate velocity $a^{\star}$.

Proof. Here, we consider a sequence $\left(U_{i}^{n}\right)_{i \in \mathbb{Z}}$ defined in $\Omega$, to study the updated sequence $\left(U_{i}^{n+1}\right)_{i \in \mathbb{Z}}$ given by (2.5)-(2.6). Since $\Omega \subset \omega$, we have

$$
\rho_{\mathcal{R}}^{\Delta x}\left(\frac{x-x_{i+\frac{1}{2}}}{\Delta t} ; U_{i}^{n}, U_{i+1}^{n}\right)>0 \quad \text { and } \quad(\rho E)_{\mathcal{R}}^{\Delta x}\left(\frac{x-x_{i+\frac{1}{2}}}{\Delta t} ; U_{i}^{n}, U_{i+1}^{n}\right)>0 .
$$

By definition of $U_{i}^{n+1}$, given by (2.3), we immediately deduce that the scheme (2.5)(2.6) is weakly robust and thus we have $U_{i}^{n+1}$ in $\omega$.

Next, we prove the entropy preserving property by applying Theorem 2.5. Indeed, 
let us set $S(U)=\rho f(s(\tau, e))$ to write

$$
\begin{aligned}
& \frac{1}{\Delta x} \int_{-\frac{\Delta x}{2}}^{\frac{\Delta x}{2}} S\left(U_{\mathcal{R}}^{\Delta x}\left(\frac{x}{\Delta t} ; U_{L}, U_{R}\right)\right) d x= \\
& \frac{1}{\Delta x} \int_{-\frac{\Delta x}{2}}^{a^{-} \Delta t} \rho_{L} f\left(s\left(\tau_{L}, e_{L}\right)\right) d x+\frac{1}{\Delta x} \int_{-a^{-} \Delta t}^{a^{\star} \Delta t}\left\{\rho_{L}^{\star} f\left(s\left(\tau_{L}^{\star}, e_{L}^{\star}\right)\right)\right\}\left(\frac{x}{\Delta t} ; U_{L}, U_{R}\right) d x \\
& +\frac{1}{\Delta x} \int_{-a^{\star} \Delta t}^{a^{+} \Delta t}\left\{\rho_{R}^{\star} f\left(s\left(\tau_{R}^{\star}, e_{R}^{\star}\right)\right)\right\}\left(\frac{x}{\Delta t} ; U_{L}, U_{R}\right) d x+\frac{1}{\Delta x} \int_{a^{+} \Delta t}^{\frac{\Delta x}{2}} \rho_{R} f\left(s\left(\tau_{R}, e_{R}\right)\right) d x .
\end{aligned}
$$

Let us assume the function $U \mapsto S(U)$ to be convex so that $f$ is a decreasing function. By involving the local entropy minimum principle (3.3), we get

$$
\begin{array}{ll}
f\left(s\left(\tau_{L}^{\star}, e_{L}^{\star}\right)\right)\left(\frac{x}{\Delta t} ; U_{L}, U_{R}\right) \leq f\left(s\left(\tau_{L}, e_{L}\right)\right), & \text { if } \frac{x}{t} \in\left(a^{-}, a^{\star}\right), \\
f\left(s\left(\tau_{R}^{\star}, e_{R}^{\star}\right)\right)\left(\frac{x}{\Delta t} ; U_{L}, U_{R}\right) \leq f\left(s\left(\tau_{R}, e_{R}\right)\right), & \text { if } \frac{x}{t} \in\left(a^{\star}, a^{+}\right) .
\end{array}
$$

Hence, we can rewrite (3.5) as follows:

$$
\begin{aligned}
\frac{1}{\Delta x} \int_{-\frac{\Delta x}{2}}^{\frac{\Delta x}{2}} S\left(U_{\mathcal{R}}^{\Delta x}\left(\frac{x}{\Delta t} ; U_{L}, U_{R}\right)\right) d x \leq & \frac{f\left(s\left(\tau_{L}, e_{L}\right)\right)}{\Delta x} \int_{-\frac{\Delta x}{2}}^{a^{\star} \Delta t} \rho_{\mathcal{R}}^{\Delta x}\left(\frac{x}{\Delta t} ; U_{L}, U_{R}\right) d x \\
& +\frac{f\left(s\left(\tau_{R}, e_{R}\right)\right)}{\Delta x} \int_{a^{\star} \Delta t}^{\frac{\Delta x}{2}} \rho_{\mathcal{R}}^{\Delta x}\left(\frac{x}{\Delta t} ; U_{L}, U_{R}\right) d x .
\end{aligned}
$$

Let us plug the half consistency conditions (3.2) and (3.4) into the above relation to obtain:

$$
\begin{aligned}
\frac{1}{\Delta x} \int_{-\frac{\Delta x}{2}}^{\frac{\Delta x}{2}} S\left(U_{\mathcal{R}}^{\Delta x}\left(\frac{x}{\Delta t} ; U_{L}, U_{R}\right)\right) d x \leq \\
\frac{1}{2}\left(S\left(U_{L}\right)+S\left(U_{R}\right)\right)-\frac{\Delta t}{\Delta x}\left(S\left(U_{R}\right) u_{R}-S\left(U_{L}\right) u_{L}\right) .
\end{aligned}
$$

From Theorem 2.5, we immediately deduce the expected entropy preserving property.

Next, let us establish the entropy maximum principle property. By the well-known Jensen inequality, we have

$$
\begin{aligned}
\rho_{i}^{n+1} f\left(s\left(\tau_{i}^{n+1}, e_{i}^{n+1}\right)\right) \leq & \frac{1}{\Delta x} \int_{x_{i-\frac{1}{2}}}^{x_{i+\frac{1}{2}}} S\left(U^{\Delta x}\left(x, t^{n}+\Delta t\right)\right) d x \\
\leq & \frac{1}{\Delta x} \int_{x_{i-\frac{1}{2}}}^{x_{i}} S\left(U_{\mathcal{R}}^{\Delta x}\left(\frac{x-x_{i-\frac{1}{2}}}{\Delta t} ; U_{i-1}^{n}, U_{i}^{n}\right)\right) d x \\
& +\frac{1}{\Delta x} \int_{x_{i}}^{x_{i+\frac{1}{2}}} S\left(U_{\mathcal{R}}^{\Delta x}\left(\frac{x-x_{i+\frac{1}{2}}}{\Delta t} ; U_{i}^{n}, U_{i+1}^{n}\right)\right) d x .
\end{aligned}
$$

Since $\rho_{\mathcal{R}}^{\Delta x}\left(\frac{x}{\Delta t} ; U_{L}, U_{R}\right)>0$ and $f$ monoton decreasing, we have

$$
\begin{aligned}
& S\left(U_{\mathcal{R}}^{\Delta x}\left(\frac{x-x_{i+\frac{1}{2}}}{\Delta t} ; U_{i}^{n}, U_{i+1}^{n}\right)\right) \leq \\
& \quad \rho_{\mathcal{R}}^{\Delta x}\left(\frac{x-x_{i+\frac{1}{2}}}{\Delta t} ; U_{i}^{n}, U_{i+1}^{n}\right) f\left(\min \left(s\left(\tau_{i}^{n}, e_{i}^{n}\right), s\left(\tau_{i+1}^{n}, e_{i+1}^{n}\right)\right) .\right.
\end{aligned}
$$


As a consequence, we immediately obtain

$$
\begin{aligned}
\rho_{i}^{n+1} f\left(s\left(\tau_{i}^{n+1}, e_{i}^{n+1}\right)\right) & \leq \\
& \frac{1}{\Delta x} f\left(\min \left(s\left(\tau_{i-1}^{n}, e_{i-1}^{n}\right), s\left(\tau_{i}^{n}, e_{i}^{n}\right), s\left(\tau_{i+1}^{n}, e_{i+1}^{n}\right)\right) \int_{x_{i-\frac{1}{2}}}^{x_{i+\frac{1}{2}}} \rho^{\Delta x}\left(x, t^{n}+\Delta t\right) d x,\right.
\end{aligned}
$$

which gives the expected entropy minimum principle (2.7).

Now, the monoton property (1.4), satisfied by the function $e \mapsto s(\tau, e), \tau>0$ fixed, associated with the asymptotic assumption (1.5) implies $e_{i}^{n+1}>0$ and then we have $U_{i}^{n} \in \Omega$ for all $i$ in $\mathbb{Z}$. The proof is achieved.

Let us emphasize that the above result is more restrictive than Harten-Lax Theorem 2.5. Some entropy preserving schemes do not enter the proposed framework. For instance, Theorem 3.1 can not be applied when considering the one constant intermediate state Riemann solver, namely the HLL Riemann approximation. Indeed, with an intermediate state given by [21]:

$$
U^{\star}\left(\frac{x}{t} ; U_{L}, U_{R}\right)=\frac{a^{+} U_{R}-a^{-} U_{L}}{a^{+}-a^{-}}-\frac{1}{a^{+}-a^{-}}\left(F\left(U_{L}\right)-F\left(U_{R}\right)\right),
$$

it is not possible to ensure simultaneously

$$
s\left(\tau^{\star}, e^{\star}\right) \geq s\left(\tau_{L}, e_{L}\right) \quad \text { and } \quad s\left(\tau^{\star}, e^{\star}\right) \geq s\left(\tau_{R}, e_{R}\right) .
$$

However, many schemes enter the proposed approach. For instance, the HLLC schemes and the Suliciu relaxation schemes are relevant candidate. In $[7,6,3,1,10]$, results similar to Theorem 3.1 can be found but specified for a given scheme. These two scheme families will be consider later on. Several exemples will be detailed in the next section.

Now, our main objective concerns the derivation of suitable conditions to satisfy the local entropy minimum principle (3.3). Following ideas introduced by Chalons [10] (see also [7, 3]), we will introduce some abstract functions to be invariant on each side $\left(a^{-}, a^{\star}\right)$ and $\left(a^{\star}, a^{+}\right)$of the approximate Riemann solver. Enforcing a relevant choice of these invariant functions, we will obtain a minimum principle to get the expected relations (3.3).

To address such an issue, we need some technical results. First, we introduce two distinct functions $(\tau, e, \alpha) \mapsto \varphi(\tau, e, \alpha)$ and $(\tau, e, \alpha) \mapsto \phi(\tau, e, \alpha)$ in $C^{2}\left(\mathbb{R}^{+} \times \mathbb{R}^{+} \times \mathbb{R}, \mathbb{R}\right)$ and a smooth function $(\tau, e) \mapsto \pi(\tau, e)$ in $C^{1}\left(\mathbb{R}^{+} \times \mathbb{R}^{+}, \mathbb{R}\right)$. These functions are now assumed to satisfy several restrictions. For the sake of clarity in the presentation, we denote $\Sigma=(\tau, e, \alpha)^{T} \in \mathbb{R}^{+} \times \mathbb{R}^{+} \times \mathbb{R}$ and we set $\Sigma^{e q}=(\tau, e, \pi(\tau, e))^{T}$. In addition, we denote $\sigma=(\tau, e)^{T}$.

Next, in order to shorten the notations, it will be helpful to introduce the following functions:

$$
\begin{aligned}
D(\sigma)= & \left(\frac{\partial \varphi}{\partial \tau}\left(\Sigma^{e q}\right)+\frac{\partial \pi}{\partial \tau}(\sigma) \frac{\partial \varphi}{\partial \alpha}\left(\Sigma^{e q}\right)\right)\left(\frac{\partial \phi}{\partial e}\left(\Sigma^{e q}\right)+\frac{\partial \pi}{\partial e}(\sigma) \frac{\partial \phi}{\partial \alpha}\left(\Sigma^{e q}\right)\right) \\
& -\left(\frac{\partial \phi}{\partial \tau}\left(\Sigma^{e q}\right)+\frac{\partial \pi}{\partial \tau}(\sigma) \frac{\partial \phi}{\partial \alpha}\left(\Sigma^{e q}\right)\right)\left(\frac{\partial \varphi}{\partial e}\left(\Sigma^{e q}\right)+\frac{\partial \pi}{\partial e}(\sigma) \frac{\partial \varphi}{\partial \alpha}\left(\Sigma^{e q}\right)\right),
\end{aligned}
$$

and

$$
J(\Sigma)=p(\sigma)\left(\frac{\partial \varphi}{\partial \alpha}(\Sigma) \frac{\partial \phi}{\partial e}(\Sigma)-\frac{\partial \phi}{\partial \alpha}(\Sigma) \frac{\partial \varphi}{\partial e}(\Sigma)\right)-\left(\frac{\partial \varphi}{\partial \alpha}(\Sigma) \frac{\partial \phi}{\partial \tau}(\Sigma)-\frac{\partial \phi}{\partial \alpha}(\Sigma) \frac{\partial \varphi}{\partial \tau}(\Sigma)\right) .
$$


In addition, we here denote

$$
\nabla_{\Sigma} \varphi(\Sigma)=\left(\frac{\partial \varphi}{\partial \tau}, \frac{\partial \varphi}{\partial e}, \frac{\partial \varphi}{\partial \alpha}\right)^{T}
$$

to set for all pairs $\left(\Sigma_{1}, \Sigma_{2}\right)$ :

$$
\begin{aligned}
\mathcal{H}\left(\Sigma_{1}, \Sigma_{2}\right)=\frac{\partial^{2} \varphi}{\partial \alpha^{2}}\left(\Sigma_{2}\right) \frac{\partial \phi}{\partial \alpha}\left(\Sigma_{1}\right)-\frac{\partial^{2} \phi}{\partial \alpha^{2}}\left(\Sigma_{2}\right) \frac{\partial \varphi}{\partial \alpha}\left(\Sigma_{1}\right) \\
+\gamma\left(\Sigma_{1}, \Sigma_{2}\right)\left(\frac{\partial \varphi}{\partial \alpha}\left(\Sigma_{2}\right) \frac{\partial^{2} \phi}{\partial \alpha^{2}}\left(\Sigma_{1}\right)-\frac{\partial \phi}{\partial \alpha}\left(\Sigma_{2}\right) \frac{\partial^{2} \varphi}{\partial \alpha^{2}}\left(\Sigma_{1}\right)\right) \\
+\frac{\gamma\left(\Sigma_{1}, \Sigma_{2}\right)}{D\left(\sigma_{1}\right)}\left(\frac{\partial \varphi}{\partial \alpha}\left(\Sigma_{2}\right) \nabla_{\Sigma}\left(\frac{\partial \phi}{\partial \alpha}\right)\left(\Sigma_{1}\right)-\frac{\partial \phi}{\partial \alpha}\left(\Sigma_{2}\right) \nabla_{\Sigma}\left(\frac{\partial \varphi}{\partial \alpha}\right)\left(\Sigma_{1}\right)\right) \\
\cdot\left(\nabla_{\Sigma} \phi \wedge \nabla_{\Sigma} \varphi\right)\left(\Sigma_{1}\right),
\end{aligned}
$$

where

$$
\gamma\left(\Sigma_{1}, \Sigma_{2}\right)=\frac{\frac{\partial \varphi}{\partial \alpha}\left(\Sigma_{2}\right)}{\frac{\partial \varphi}{\partial \alpha}\left(\Sigma_{1}\right)} \quad \text { with } \frac{\partial \varphi}{\partial \alpha}\left(\Sigma_{1}\right) \neq 0
$$

Finally, we denote

$$
K(\Sigma)=\frac{\partial \varphi}{\partial e}(\Sigma) \frac{\partial \phi}{\partial \alpha}(\Sigma)-\frac{\partial \varphi}{\partial \alpha}(\Sigma) \frac{\partial \phi}{\partial e}(\Sigma) .
$$

We now give our central technical statement.

Proposition 3.2. Consider two functions $(\tau, e, \alpha) \mapsto \varphi(\tau, e, \alpha)$ and $(\tau, e, \alpha) \mapsto$ $\phi(\tau, e, \alpha)$ in $C^{2}\left(\mathbb{R}^{+} \times \mathbb{R}^{+} \times \mathbb{R}, \mathbb{R}\right)$ and a function $(\tau, e) \mapsto \pi(\tau, e)$ in $C^{1}\left(\mathbb{R}^{+} \times \mathbb{R}^{+}, \mathbb{R}\right)$ so that the following conditions are satisfied for all $(\tau, e)$ under consideration:

(i) $D(\tau, e) \neq 0$ where the function $D$ is defined by (3.6),

(ii) $J(\tau, e, \pi(\tau, e))=0$ where the function $J$ is defined by (3.7). Moreover, we assume

(iii) for fixed $(\tau, e)$, the function $\alpha \mapsto \varphi(\tau, e, \alpha)$ is strictly monoton and, for all pairs $\left(\Sigma_{1}, \Sigma_{2}\right)$ in $\left(\mathbb{R}^{+} \times \mathbb{R}^{+} \times \mathbb{R}\right)^{2}$, we have $K\left(\Sigma_{1}\right) \mathcal{H}\left(\Sigma_{1}, \Sigma_{2}\right)<0$ where $K$ and $\mathcal{H}$ are respectively defined by (3.10) and (3.8).

Then there exists a function $(\tau, e, \alpha) \mapsto \mathcal{S}(\varphi(\tau, e, \alpha), \phi(\tau, e, \alpha))$ so that

$$
\max _{\alpha \in \mathbb{R}} \mathcal{S}(\varphi(\tau, e, \alpha), \phi(\tau, e, \alpha))=\left.\mathcal{S}(\varphi(\tau, e, \alpha), \phi(\tau, e, \alpha))\right|_{\alpha=\pi(\tau, e)}=s(\tau, e)
$$

where $s(\tau, e)$ is nothing but the specific entropy.

Before we give the proof of this result, let us illustrate the interest of this technical proposition. Indeed, by assuming the pair $(\varphi, \phi)$ to be invariant by the approximate Riemann solver (2.1) on the half domain defined defined by $\frac{x}{t} \leq a^{\star}$ or $\frac{x}{t} \geq a^{\star}$, we are able to ensure the entropy maximum principle (3.3).

THEOREM 3.3. Let $U_{L}$ and $U_{R}$ be two given constant states in $\Omega$. Consider $U_{\mathcal{R}}^{\Delta x}\left(\frac{x}{t} ; U_{L}, U_{R}\right) \in \omega$. Assume the existence of $a^{\star} \in\left(a^{-}, a^{+}\right)$to obtain the decomposition (3.1) of the intermediate state in (2.1) and assume that the consistency condition (2.4) and the half consistency condition (3.2) are satisfied.

Assume the existence of smooth functions $\varphi: \mathbb{R}^{+} \times \mathbb{R}^{+} \times \mathbb{R} \rightarrow \mathbb{R}, \phi: \mathbb{R}^{+} \times \mathbb{R}^{+} \times \mathbb{R} \rightarrow$ $\mathbb{R}$ and $\pi: \mathbb{R}^{+} \times \mathbb{R}^{+} \rightarrow \mathbb{R}$, and a function $\left(\frac{x}{t} ; U_{L}, U_{R}\right) \mapsto \alpha^{\star}\left(\frac{x}{t} ; U_{L}, U_{R}\right)$ such that, for 
all $\frac{x}{t} \in\left(a^{-}, a^{\star}\right)$, we have

$$
\begin{aligned}
& \varphi\left(\tau_{L}, e_{L}, \pi\left(\tau_{L}, e_{L}\right)\right)=\varphi\left(\tau_{L}^{\star}, e_{L}^{\star}, \alpha^{\star}\right)\left(\frac{x}{t} ; U_{L}, U_{R}\right), \\
& \phi\left(\tau_{L}, e_{L}, \pi\left(\tau_{L}, e_{L}\right)\right)=\phi\left(\tau_{L}^{\star}, e_{L}^{\star}, \alpha^{\star}\right)\left(\frac{x}{t} ; U_{L}, U_{R}\right),
\end{aligned}
$$

respectively for all $\frac{x}{t} \in\left(a^{\star}, a^{+}\right)$:

$$
\begin{aligned}
& \varphi\left(\tau_{R}, e_{R}, \pi\left(\tau_{R}, e_{R}\right)\right)=\varphi\left(\tau_{R}^{\star}, e_{R}^{\star}, \alpha^{\star}\right)\left(\frac{x}{t} ; U_{L}, U_{R}\right) \\
& \phi\left(\tau_{R}, e_{R}, \pi\left(\tau_{R}, e_{R}\right)\right)=\phi\left(\tau_{R}^{\star}, e_{R}^{\star}, \alpha^{\star}\right)\left(\frac{x}{t} ; U_{L}, U_{R}\right) .
\end{aligned}
$$

Then, as soon as the triple $(\varphi, \phi, \pi)$ satisfies the assumptions (i)-(ii)-(iii) Proposition 3.2, the left (respect. right) local maximum principle (3.3a) (respect. (3.3b) is verified. As a consequence the scheme (2.5)-(2.6) is robust, entropy preserving and entropy maximum principle preserving.

Proof. Since the establishment of each local maximum principle are similar, we here focus on (3.3a). First, we note that Proposition 3.2 can be applied and thus there exists a function $(\tau, e, \alpha) \mapsto \mathcal{S}(\tau, e, \alpha)$ such that the relation (3.11) holds. As a consequence, for all $\frac{x}{t}$ fixed in $\left(a^{-}, a^{\star}\right)$, we have

$$
\begin{aligned}
s\left(\tau_{L}^{\star}, e_{L}^{\star}\right)\left(\frac{x}{t} ; U_{L}, U_{R}\right) & =\max _{\alpha \in \mathbb{R}} \mathcal{S}\left(\varphi\left(\tau_{L}^{\star}, e_{L}^{\star}, \alpha\right), \phi\left(\tau_{L}^{\star}, e_{L}^{\star}, \alpha\right)\right)\left(\frac{x}{t} ; U_{L}, U_{R}\right), \\
& \geq \mathcal{S}\left(\varphi\left(\tau_{L}^{\star}, e_{L}^{\star}, \beta\right), \phi\left(\tau_{L}^{\star}, e_{L}^{\star}, \beta\right)\right)\left(\frac{x}{t} ; U_{L}, U_{R}\right), \quad \forall \beta \in \mathbb{R} .
\end{aligned}
$$

We fix $\beta=\alpha^{\star}\left(\frac{x}{t} ; U_{L}, U_{R}\right)$ to write

$$
s\left(\tau_{L}^{\star}, e_{L}^{\star}\right)\left(\frac{x}{t} ; U_{L}, U_{R}\right) \geq \mathcal{S}\left(\varphi\left(\tau_{L}^{\star}, e_{L}^{\star}, \alpha^{\star}\right), \phi\left(\tau_{L}^{\star}, e_{L}^{\star}, \alpha^{\star}\right)\right)\left(\frac{x}{t} ; U_{L}, U_{R}\right) .
$$

Next, by involving the invariant principle (3.12)-(3.13), we get

$$
s\left(\tau_{L}^{\star}, e_{L}^{\star}\right)\left(\frac{x}{t} ; U_{L}, U_{R}\right) \geq \mathcal{S}\left(\varphi\left(\tau_{L}, e_{L}, \pi\left(\tau_{L}, e_{L}\right)\right), \phi\left(\tau_{L}, e_{L}, \pi\left(\tau_{L}, e_{L}\right)\right)\right) .
$$

Since, by definition of $\mathcal{S}$ we have

$$
s\left(\tau_{L}, e_{L}\right)=\mathcal{S}\left(\varphi\left(\tau_{L}, e_{L}, \alpha\right),\left.\phi\left(\tau_{L}, e_{L}, \alpha\right)\right|_{\alpha=\pi\left(\tau_{L}, e_{L}\right)},\right.
$$

the expected left minimum principle (3.3a) is reached and the proof is completed.

To complete the present section, we now establish Proposition 3.2. To access such an issue, we need the following three lemmas whose helpfulness is just technical. In the sequel, we systematicaly denote $(\tau, e, \alpha) \mapsto \varphi(\tau, e, \alpha)$ and $(\tau, e, \alpha) \mapsto \phi(\tau, e, \alpha)$ two smooth functions in $C^{2}\left(\mathbb{R}^{+} \times \mathbb{R}^{+} \times \mathbb{R}, \mathbb{R}\right)$ and $(\tau, e) \mapsto \pi(\tau, e)$ in $C^{1}\left(\mathbb{R}^{+} \times \mathbb{R}^{+}, \mathbb{R}\right)$.

Lemma 3.4. Let $D(\tau, e): \mathbb{R}^{+} \times \mathbb{R}^{+} \rightarrow \mathbb{R}$ be defined by (3.6). Assume $D(\tau, e) \neq 0$ for all $(\tau, e)$ under consideration. Then there exists two functions, denoted $\bar{\tau}(X, Y)$ and $\bar{e}(X, Y)$, such that

$$
\bar{\tau}\left(\varphi\left(\Sigma^{e q}\right), \phi\left(\Sigma^{e q}\right)\right)=\tau \quad \text { and } \quad \bar{e}\left(\varphi\left(\Sigma^{e q}\right), \phi\left(\Sigma^{e q}\right)\right)=e,
$$


where $\Sigma^{e q}=(\tau, e, \pi(\tau, e))^{T}$, and the following derivatives are satisfied:

$$
\begin{aligned}
& \frac{\partial \bar{\tau}}{\partial X}(X, Y)=\frac{1}{D(\tilde{\sigma})}\left(\frac{\partial \phi}{\partial e}\left(\tilde{\Sigma}^{e q}\right)+\frac{\partial \pi}{\partial e}(\bar{\sigma}) \frac{\partial \phi}{\partial \alpha}\left(\tilde{\Sigma}^{e q}\right)\right), \\
& \frac{\partial \bar{\tau}}{\partial Y}(X, Y)=-\frac{1}{D(\tilde{\sigma})}\left(\frac{\partial \varphi}{\partial e}\left(\tilde{\Sigma}^{e q}\right)+\frac{\partial \pi}{\partial e}(\bar{\sigma}) \frac{\partial \varphi}{\partial \alpha}\left(\tilde{\Sigma}^{e q}\right)\right), \\
& \frac{\partial \bar{e}}{\partial X}(X, Y)=-\frac{1}{D(\tilde{\sigma})}\left(\frac{\partial \phi}{\partial \tau}\left(\tilde{\Sigma}^{e q}\right)+\frac{\partial \pi}{\partial \tau}(\bar{\sigma}) \frac{\partial \phi}{\partial \alpha}\left(\tilde{\Sigma}^{e q}\right)\right), \\
& \frac{\partial \bar{e}}{\partial Y}(X, Y)=\frac{1}{D(\tilde{\sigma})}\left(\frac{\partial \varphi}{\partial \tau}\left(\tilde{\Sigma}^{e q}\right)+\frac{\partial \pi}{\partial \tau}(\bar{\sigma}) \frac{\partial \varphi}{\partial \alpha}\left(\tilde{\Sigma}^{e q}\right)\right),
\end{aligned}
$$

where we have set

$$
\tilde{\sigma}=(\bar{\tau}(X, Y), \bar{e}(X, Y))^{T} \quad \text { and } \quad \tilde{\Sigma}^{e q}=(\bar{\tau}(X, Y), \bar{e}(X, Y), \pi(\bar{\tau}(X, Y), \bar{e}(X, Y)))^{T} .
$$

In the second statement, we exhibit a suitable function derived from $\bar{\tau}$ and $\bar{e}$ and we consider its local extremum.

Lemma 3.5. Let $D(\tau, e): \mathbb{R}^{+} \times \mathbb{R}^{+} \rightarrow \mathbb{R}$ be defined by (3.6) and $J(\tau, e, \alpha)$ : $\mathbb{R}^{+} \times \mathbb{R}^{+} \times \mathbb{R} \rightarrow \mathbb{R}$ be defined by (3.7). For all $(\tau, e)$ under consideration, we assume $D(\tau, e) \neq 0$ and $J(\tau, e, \pi(\tau, e))=0$. We introduce $S(\tau, e, \alpha): \mathbb{R}^{+} \times \mathbb{R}^{+} \times \mathbb{R} \rightarrow \mathbb{R}$ defined by:

$$
S(\tau, e, \alpha)=s(\bar{\tau}(\varphi(\Sigma), \phi(\Sigma)), \bar{e}(\varphi(\Sigma), \phi(\Sigma)))
$$

where we have set $\Sigma=(\tau, e, \alpha)^{T}$ and $s(\tau, e)$ denotes the specific entropy. Then we have

$$
\frac{\partial \varphi}{\partial e}\left(\bar{\Sigma}^{e q}\right) \frac{\partial \phi}{\partial \alpha}\left(\bar{\Sigma}^{e q}\right)-\frac{\partial \varphi}{\partial \alpha}\left(\bar{\Sigma}^{e q}\right) \frac{\partial \phi}{\partial e}\left(\bar{\Sigma}^{e q}\right) \neq 0
$$

and

$$
\frac{\partial S}{\partial \alpha}=\frac{1}{T(\bar{\sigma})} \frac{\frac{\partial \varphi}{\partial \alpha}(\Sigma) \frac{\partial \phi}{\partial \alpha}\left(\bar{\Sigma}^{e q}\right)-\frac{\partial \varphi}{\partial \alpha}\left(\bar{\Sigma}^{e q}\right) \frac{\partial \phi}{\partial \alpha}\left(\bar{\Sigma}^{e q}\right)-\frac{\partial \phi}{\partial \alpha}(\Sigma)}{\partial \alpha}\left(\bar{\Sigma}^{e q}\right) \frac{\partial \phi}{\partial e}\left(\bar{\Sigma}^{e q}\right),
$$

where

$$
\begin{aligned}
& \bar{\sigma}=(\bar{\tau}(\varphi(\Sigma), \phi(\Sigma)), \bar{e}(\varphi(\Sigma), \phi(\Sigma)))^{T}, \\
& \bar{\Sigma}^{e q}=(\bar{\tau}(\varphi(\Sigma), \phi(\Sigma)), \bar{e}(\varphi(\Sigma), \phi(\Sigma)), \pi(\bar{\tau}(\varphi(\Sigma), \phi(\Sigma)), \bar{e}(\varphi(\Sigma), \phi(\Sigma))))^{T},
\end{aligned}
$$

with the functions $\bar{\tau}$ and $\bar{e}$ defined in Lemma 3.4. Here, the function $T(\tau, e)>0$ denotes the temperature according to the law (1.3).

The last result concerns the study of the extrema of the function $S$, defined by (3.22), by solving in $\alpha$ the following equation:

$$
\frac{\partial \varphi}{\partial \alpha}(\Sigma) \frac{\partial \phi}{\partial \alpha}\left(\bar{\Sigma}^{e q}\right)-\frac{\partial \varphi}{\partial \alpha}\left(\bar{\Sigma}^{e q}\right) \frac{\partial \phi}{\partial \alpha}(\Sigma)=0 .
$$

Lemma 3.6. Let $D(\tau, e): \mathbb{R}^{+} \times \mathbb{R}^{+} \rightarrow \mathbb{R}$ be defined by (3.6) and $\mathcal{H}\left(\Sigma_{1}, \Sigma_{2}\right)$ : $\left(\mathbb{R}^{+} \times \mathbb{R}^{+} \times \mathbb{R}\right)^{2} \rightarrow \mathbb{R}$ be defined by (3.8). Assume, for all $(\tau, e)$ under consideration, $D(\tau, e) \neq 0$ and $\mathcal{H}\left(\Sigma_{1}, \Sigma_{2}\right) \neq 0$ for all $\left(\Sigma_{1}, \Sigma_{2}\right) \in\left(\mathbb{R}^{+} \times \mathbb{R}^{+} \times \mathbb{R}\right)^{2}$. In addition, 
assume that the function $\alpha \mapsto \varphi(\tau, e, \alpha)$ is strictly monoton. Then the equation (3.25) admits a unique solution given by $\alpha=\pi(\tau, e)$.

Equipped with these results, we can establish Proposition 3.2.

Proof. [Proof of Proposition 3.2] From assumption (i) and (ii), we can apply Lemmas 3.4 and 3.5 to define a function $\mathcal{S}$ as follows:

$$
\begin{aligned}
\mathcal{S}(\varphi(\Sigma), \phi(\Sigma)) & =S(\Sigma) \\
& =s(\bar{\tau}(\varphi(\Sigma), \phi(\Sigma)), \bar{e}(\varphi(\Sigma), \phi(\Sigma)))
\end{aligned}
$$

where the function $s$ is nothing but the specific entropy. Now, by definition of $\bar{\tau}$ and $\bar{e}$, we have

$$
\left.\bar{\tau}(\varphi(\Sigma), \phi(\Sigma))\right|_{\alpha=\pi(\tau, e)}=\tau \quad \text { and }\left.\quad \bar{e}(\varphi(\Sigma), \phi(\Sigma))\right|_{\alpha=\pi(\tau, e)}=e
$$

which immediately implies

$$
\left.\mathcal{S}(\varphi(\Sigma), \phi(\Sigma))\right|_{\alpha=\pi(\tau, e)}=s(\tau, e)
$$

Next, we study the extrema of the function $\alpha \mapsto S(\Sigma)$ that are characterized by $\frac{\partial S}{\partial \alpha}(\Sigma)=0$. Once again by Lemma 3.5, these extrema are thus determined as solutions of (3.25). But, from assumption (iii), Lemma 3.6 ensures the existence and uniqueness of the equation (3.25) and the solution is given by $\alpha=\pi(\tau, e)$.

As a consequence, we know that the function $\alpha \mapsto S(\Sigma)$ admits a global extremum located at $\alpha=\pi(\tau, e)$. In addtion, we have $\left.S(\Sigma)\right|_{\alpha=\pi(\tau, e)}=s(\tau, e)$. The proof will be completed as soon as the extremum will be proved to be minimum. To address such an issue, we evaluate $\left.\frac{\partial^{2} S}{\partial \alpha^{2}}(\Sigma)\right|_{\alpha=\pi(\tau, e)}$.

To simplify the notations, we set

$$
g(\tau, e, \alpha)=\frac{\partial \phi}{\partial \alpha}(\Sigma) \frac{\partial \varphi}{\partial \alpha}\left(\bar{\Sigma}^{e q}\right)-\frac{\partial \phi}{\partial \alpha}\left(\bar{\Sigma}^{e q}\right) \frac{\partial \varphi}{\partial \alpha}(\Sigma)
$$

with $\bar{\Sigma}^{e q}$ given by (3.24), to write

$$
\frac{\partial S}{\partial \alpha}(\tau, e, \alpha)=\frac{1}{T(\bar{\sigma})} \frac{g(\tau, e, \alpha)}{K\left(\bar{\Sigma}^{e q}\right)},
$$

where $K$ is defined by (3.10) and never vanishes.

Since, for all $(\tau, e)$ under consideration, we have $T(\tau, e)>0$ and $g(\tau, e, \pi(\tau, e))=$ 0, we obtain:

$$
\frac{\partial^{2} S}{\partial \alpha^{2}}(\tau, e, \pi(\tau, e))=\frac{1}{T(\sigma)} \frac{g^{\prime}(\tau, e, \pi(\tau, e))}{K\left(\Sigma^{e q}\right)},
$$

where $g^{\prime}$ is the derivative of $g$ with respect to $\alpha$. We have set $\Sigma^{e q}=(\tau, e, \pi(\tau, e))^{T}$ for clarity in the notations. 
We now compute $g^{\prime}$ as follows:

$$
\begin{aligned}
& g^{\prime}\left(\Sigma^{e q}\right)=\frac{\partial^{2} \phi}{\partial \alpha^{2}}\left(\Sigma^{e q}\right) \frac{\partial \varphi}{\partial \alpha}\left(\Sigma^{e q}\right)-\frac{\partial^{2} \varphi}{\partial \alpha^{2}}\left(\Sigma^{e q}\right) \frac{\partial \phi}{\partial \alpha}\left(\Sigma^{e q}\right) \\
& +\frac{\partial \phi}{\partial \alpha}\left(\Sigma^{e q}\right)\left(\begin{array}{r}
\frac{\partial^{2} \varphi}{\partial \tau \partial \alpha}\left(\Sigma^{e q}\right) \frac{\partial \bar{\tau}(\varphi(\Sigma), \phi(\Sigma))}{\partial \alpha}\left(\Sigma^{e q}\right)+\frac{\partial^{2} \varphi}{\partial e \partial \alpha}\left(\Sigma^{e q}\right) \frac{\partial \bar{e}(\varphi(\Sigma), \phi(\Sigma))}{\partial \alpha}\left(\Sigma^{e q}\right) \\
+\frac{\partial^{2} \varphi}{\partial \alpha^{2}}\left(\Sigma^{e q}\right) \frac{\partial \pi(\bar{\tau}(\varphi(\Sigma), \phi(\Sigma)), \bar{e}(\varphi(\Sigma), \phi(\Sigma)))}{\partial \alpha}\left(\Sigma^{e q}\right)
\end{array}\right) \\
& -\frac{\partial \varphi}{\partial \alpha}\left(\Sigma^{e q}\right)\left(\begin{array}{r}
\frac{\partial^{2} \phi}{\partial \tau \partial \alpha}\left(\Sigma^{e q}\right) \frac{\partial \bar{\tau}(\varphi(\Sigma), \phi(\Sigma))}{\partial \alpha}\left(\Sigma^{e q}\right)+\frac{\partial^{2} \phi}{\partial e \partial \alpha}\left(\Sigma^{e q}\right) \frac{\partial \bar{e}(\varphi(\Sigma), \phi(\Sigma))}{\partial \alpha}\left(\Sigma^{e q}\right) \\
+\frac{\partial^{2} \phi}{\partial \alpha^{2}}\left(\Sigma^{e q}\right) \frac{\partial \pi(\bar{\tau}(\varphi), \phi(\Sigma)), \bar{e}(\varphi(\Sigma), \phi(\Sigma)))}{\partial \alpha}\left(\Sigma^{e q}\right)
\end{array}\right)
\end{aligned}
$$

Arguing the derivative formulas (3.17)-(3.20), a straightforward computation yields

$$
\begin{aligned}
& \frac{\partial \bar{\tau}(\varphi(\Sigma), \phi(\Sigma))}{\partial \alpha}\left(\Sigma^{e q}\right)= \frac{1}{D(\tau, e)}\left(\frac{\partial \phi}{\partial e}\left(\Sigma^{e q}\right) \frac{\partial \varphi}{\partial \alpha}\left(\Sigma^{e q}\right)-\frac{\partial \varphi}{\partial e}\left(\Sigma^{e q}\right) \frac{\partial \phi}{\partial \alpha}\left(\Sigma^{e q}\right)\right) \\
& \frac{\partial \bar{e}(\varphi(\Sigma), \phi(\Sigma))}{\partial \alpha}\left(\Sigma^{e q}\right)= \frac{1}{D(\tau, e)}\left(\frac{\partial \varphi}{\partial \tau}\left(\Sigma^{e q}\right) \frac{\partial \phi}{\partial \alpha}\left(\Sigma^{e q}\right)-\frac{\partial \phi}{\partial \tau}\left(\Sigma^{e q}\right) \frac{\partial \varphi}{\partial \alpha}\left(\Sigma^{e q}\right)\right) \\
& \frac{\partial \pi(\bar{\tau}(\varphi(\Sigma), \phi(\Sigma)), \bar{e}(\varphi(\Sigma), \phi(\Sigma)))}{\partial \alpha}\left(\Sigma^{e q}\right)= \\
& 1-\frac{1}{D(\tau, e)}\left(\frac{\partial \varphi}{\partial \tau}\left(\Sigma^{e q}\right) \frac{\partial \phi}{\partial e}\left(\Sigma^{e q}\right)-\frac{\partial \phi}{\partial \tau}\left(\Sigma^{e q}\right) \frac{\partial \varphi}{\partial e}\left(\Sigma^{e q}\right)\right) .
\end{aligned}
$$

We plug these relations into (3.26) to get

$$
g^{\prime}\left(\Sigma^{e q}\right)=\mathcal{H}\left(\Sigma^{e q}, \Sigma^{e q}\right),
$$

where the function $\mathcal{H}$ is defined by (3.8). Hence, we obtain:

$$
\frac{\partial^{2} S}{\partial \alpha^{2}}(\tau, e, \pi(\tau, e))=\frac{1}{T(\sigma)} \frac{\mathcal{H}\left(\Sigma^{e q}, \Sigma^{e q}\right)}{K\left(\Sigma^{e q}\right)} .
$$

Since $\mathcal{H}\left(\Sigma^{e q}, \Sigma^{e q}\right) / K\left(\Sigma^{e q}\right)<0$ for all $(\tau, e)$ under consideration, we immediately deduce that the point $\alpha=\pi(\tau, e)$ defines the maximum of the function $\alpha \mapsto S(\tau, e, \alpha)$. The proof is achieved.

We conclude this section by giving successively the proof of the three intermediate lemmas.

Proof. [proof of Lemma 3.4] Let us consider the function $\Theta(\tau, e): \mathbb{R}^{+} \times \mathbb{R} \rightarrow \mathbb{R} \times \mathbb{R}$ and defined by

$$
\Theta(\tau, e)=\left(\begin{array}{l}
\varphi(\tau, e, \pi(\tau, e)) \\
\phi(\tau, e, \pi(\tau, e))
\end{array}\right) .
$$

We remark that the function $D(\tau, e)$, defined by (3.6), is nothing but the Jacobian function of $\Theta$. Since, for all $(\tau, e)$ under consideration, $D(\tau, e)$ does not vanish, we can apply the Implicit Theorem to deduce the existence of a reciprocal function

$$
\Theta^{-1}(X, Y)=\left(\begin{array}{c}
\bar{\tau}(X, Y) \\
\bar{e}(X, Y)
\end{array}\right),
$$


defined for $(X, Y)$ in the range of $\Theta$ and such that $D(\tau, e) \neq 0$.

By definition of the functions $\bar{\tau}$ and $\bar{e}$, the relation (3.16) is obviously obtained.

Now, we evaluate the derivative of these two reciprocal functions. Once again by definition of $\bar{\tau}$ and $\bar{e}$, we have

$$
\begin{aligned}
& \varphi(\bar{\tau}(X, Y), \bar{e}(X, Y), \pi(\bar{\tau}(X, Y), \bar{e}(X, Y)))=X \\
& \phi(\bar{\tau}(X, Y), \bar{e}(X, Y), \pi(\bar{\tau}(X, Y), \bar{e}(X, Y)))=Y .
\end{aligned}
$$

By differentiating in $X$ these two relations, we obtain:

$$
\begin{aligned}
\left(\frac{\partial \varphi}{\partial \tau}\left(\tilde{\Sigma}^{e q}\right)+\right. & \left.\frac{\partial \varphi}{\partial \alpha}\left(\tilde{\Sigma}^{e q}\right) \frac{\partial \pi}{\partial \tau}(\tilde{\sigma})\right) \frac{\partial \bar{\tau}}{\partial X}(X, Y)+ \\
& \left(\frac{\partial \varphi}{\partial e}\left(\tilde{\Sigma}^{e q}\right)+\frac{\partial \varphi}{\partial \alpha}\left(\tilde{\Sigma}^{e q}\right) \frac{\partial \pi}{\partial e}(\tilde{\sigma})\right) \frac{\partial \bar{e}}{\partial X}(X, Y)=1, \\
\left(\frac{\partial \phi}{\partial \tau}\left(\tilde{\Sigma}^{e q}\right)+\right. & \left.\frac{\partial \phi}{\partial \alpha}\left(\tilde{\Sigma}^{e q}\right) \frac{\partial \pi}{\partial \tau}(\tilde{\sigma})\right) \frac{\partial \bar{\tau}}{\partial X}(X, Y)+ \\
& \left(\frac{\partial \phi}{\partial e}\left(\tilde{\Sigma}^{e q}\right)+\frac{\partial \phi}{\partial \alpha}\left(\tilde{\Sigma}^{e q}\right) \frac{\partial \pi}{\partial e}(\tilde{\sigma})\right) \frac{\partial \bar{e}}{\partial X}(X, Y)=0,
\end{aligned}
$$

where $\tilde{\sigma}$ and $\tilde{\Sigma}^{e q}$ are given by (3.21).

With $D(\tilde{\sigma}) \neq 0$, this above $2 \times 2$ system is solvable in the variables $\left(\frac{\partial \bar{\tau}}{\partial X}, \frac{\partial \bar{e}}{\partial X}\right)$. Then we get the expected definition of the derivative $\frac{\partial \bar{\tau}}{\partial X}(X, Y)$ and $\frac{\partial \bar{e}}{\partial X}(X, Y)$ given by (3.17) and (3.19).

Similarly, the formulas (3.18) and (3.20) are obtained by differentiating (3.27) with respect to $Y$ and next by solving the resulting $2 \times 2$ system in the variables $\left(\frac{\partial \bar{\tau}}{\partial Y}, \frac{\partial \bar{e}}{\partial Y}\right)$. The proof is thus completed. $\mathrm{Q}$

Proof. [Proof of Lemma 3.5] Here, we have to compute the derivative of $S$ with respect to $\alpha$ where the function $S$ is defined by (3.22). Since $s$ denotes the specific entropy governed by (1.3), we have:

$$
\frac{\partial s}{\partial \tau}(\bar{\sigma})=\frac{p(\bar{\sigma})}{T(\bar{\sigma})} \quad \text { and } \quad \frac{\partial s}{\partial e}(\bar{\sigma})=\frac{1}{T(\bar{\sigma})},
$$

to write

$$
\begin{aligned}
\frac{\partial S}{\partial \alpha}(\Sigma)= & \frac{p(\bar{\sigma})}{T(\bar{\sigma})}\left(\frac{\partial \bar{\tau}}{\partial X}(\varphi(\Sigma), \phi(\Sigma)) \frac{\partial \varphi}{\partial \alpha}(\Sigma)+\frac{\partial \bar{\tau}}{\partial Y}(\varphi(\Sigma), \phi(\Sigma)) \frac{\partial \phi}{\partial \alpha}(\Sigma)\right) \\
& +\frac{1}{T(\bar{\sigma})}\left(\frac{\partial \bar{e}}{\partial X}(\varphi(\Sigma), \phi(\Sigma)) \frac{\partial \varphi}{\partial \alpha}(\Sigma)+\frac{\partial \bar{e}}{\partial Y}(\varphi(\Sigma), \phi(\Sigma)) \frac{\partial \phi}{\partial \alpha}(\Sigma)\right) .
\end{aligned}
$$

Next, we plug (3.17)-(3.20) into the above relation to obtain:

$$
\begin{aligned}
\frac{\partial S}{\partial \alpha}(\Sigma)=\frac{p(\bar{\sigma})}{D(\bar{\sigma}) T(\bar{\sigma})}\left[\frac{\partial \varphi}{\partial \alpha}(\Sigma)\right. & \left(\frac{\partial \phi}{\partial e}\left(\bar{\Sigma}^{e q}\right)+\frac{\partial \phi}{\partial \alpha}\left(\bar{\Sigma}^{e q}\right) \frac{\partial \pi}{\partial e}(\bar{\sigma})\right) \\
& \left.-\left(\frac{\partial \phi}{\partial \alpha}(\Sigma)\left(\frac{\partial \varphi}{\partial e}\left(\bar{\Sigma}^{e q}\right)+\frac{\partial \varphi}{\partial \alpha}\left(\bar{\Sigma}^{e q}\right) \frac{\partial \pi}{\partial e}(\bar{\sigma})\right)\right)\right] \\
+\frac{1}{D(\bar{\sigma}) T(\bar{\sigma})}\left[\frac{\partial \phi}{\partial \alpha}(\Sigma)\right. & \left(\frac{\partial \varphi}{\partial \tau}\left(\bar{\Sigma}^{e q}\right)+\frac{\partial \varphi}{\partial \alpha}\left(\bar{\Sigma}^{e q}\right) \frac{\partial \pi}{\partial \tau}(\bar{\sigma})\right) \\
& \left.-\left(\frac{\partial \varphi}{\partial \alpha}(\Sigma)\left(\frac{\partial \phi}{\partial \tau}\left(\bar{\Sigma}^{e q}\right)+\frac{\partial \phi}{\partial \alpha}\left(\bar{\Sigma}^{e q}\right) \frac{\partial \pi}{\partial \tau}(\bar{\sigma})\right)\right)\right] .
\end{aligned}
$$


Now, we remark that the condition $J\left(\Sigma^{e q}\right)=0$ with $\Sigma^{e q}=(\tau, e, \pi(\tau, e))^{T}$, implies the two following conditions:

$$
\begin{aligned}
& \frac{\partial \varphi}{\partial \alpha}\left(\Sigma^{e q}\right) \frac{\partial \phi}{\partial \tau}\left(\Sigma^{e q}\right)-\frac{\partial \phi}{\partial \alpha}\left(\Sigma^{e q}\right) \frac{\partial \varphi}{\partial \tau}\left(\Sigma^{e q}\right) \neq 0, \\
& \frac{\partial \varphi}{\partial \alpha}\left(\Sigma^{e q}\right) \frac{\partial \phi}{\partial e}\left(\Sigma^{e q}\right)-\frac{\partial \phi}{\partial \alpha}\left(\Sigma^{e q}\right) \frac{\partial \varphi}{\partial e}\left(\Sigma^{e q}\right) \neq 0 .
\end{aligned}
$$

Indeed, let us assume that one of these two relations vanishes. Then, because of $J\left(\Sigma^{e q}\right)=0$, both relations vanish. As a consequence, we obtain

$$
\begin{aligned}
& \frac{\partial \phi}{\partial \tau}\left(\Sigma^{e q}\right) \frac{\partial \varphi}{\partial \alpha}\left(\Sigma^{e q}\right)=\frac{\partial \varphi}{\partial \tau}\left(\Sigma^{e q}\right) \frac{\partial \phi}{\partial \alpha}\left(\Sigma^{e q}\right) \\
& \frac{\partial \phi}{\partial e}\left(\Sigma^{e q}\right) \frac{\partial \varphi}{\partial \alpha}\left(\Sigma^{e q}\right)=\frac{\partial \varphi}{\partial e}\left(\Sigma^{e q}\right) \frac{\partial \phi}{\partial \alpha}\left(\Sigma^{e q}\right) \\
& \frac{\partial \phi}{\partial \tau}\left(\Sigma^{e q}\right) \frac{\partial \varphi}{\partial e}\left(\Sigma^{e q}\right)=\frac{\partial \varphi}{\partial \tau}\left(\Sigma^{e q}\right) \frac{\partial \phi}{\partial e}\left(\Sigma^{e q}\right)
\end{aligned}
$$

which immediately imply $D(\tau, e)=0$ that is in contradiction with $D(\tau, e) \neq 0$ for all $(\tau, e)$ under consideration.

Arguing (3.30) and $J(\tau, e, \pi(\tau, e))=0$, we can write

$$
p(\tau, e)=\frac{\frac{\partial \varphi}{\partial \alpha}\left(\Sigma^{e q}\right) \frac{\partial \phi}{\partial \tau}\left(\Sigma^{e q}\right)-\frac{\partial \phi}{\partial \alpha}\left(\Sigma^{e q}\right) \frac{\partial \varphi}{\partial \tau}\left(\Sigma^{e q}\right)}{\frac{\partial \varphi}{\partial \alpha}\left(\Sigma^{e q}\right) \frac{\partial \phi}{\partial e}\left(\Sigma^{e q}\right)-\frac{\partial \phi}{\partial \alpha}\left(\Sigma^{e q}\right) \frac{\partial \varphi}{\partial e}\left(\Sigma^{e q}\right)} .
$$

We plug this definition of the pressure $p$ into (3.28) and a straightforward computation yields to the expected definition (3.23). The proof is thus completed.

Proof. [Proof of Lemma 3.6] Let us introduce the function $\mathcal{F}(\alpha): \mathbb{R} \rightarrow \mathbb{R}$ defined by

$$
\mathcal{F}(\alpha)=\frac{\partial \varphi}{\partial \alpha}(\Sigma) \frac{\partial \phi}{\partial \alpha}\left(\bar{\Sigma}^{e q}\right)-\frac{\partial \varphi}{\partial \alpha}\left(\bar{\Sigma}^{e q}\right) \frac{\partial \phi}{\partial \alpha}(\Sigma),
$$

where $\bar{\Sigma}^{e q}$ is given by (3.24). From now on, by definition of $\bar{\Sigma}^{e q}$, let us note that we have $\left.\bar{\Sigma}^{e q}\right|_{\alpha=\pi(\tau, e)}=(\tau, e, \pi(\tau, e))^{T}$. Then $\alpha^{0}=\pi(\tau, e)$ is a root of $\mathcal{F}$. To establish the uniqueness of $\alpha^{0}$, we assume the existence of a second root $\tilde{\alpha}^{0}$. We will show that $\mathcal{F}^{\prime}\left(\alpha^{0}\right)$ and $\mathcal{F}^{\prime}\left(\tilde{\alpha}^{0}\right)$ have the same sign which is impossible for a continuous function $\mathcal{F}$.

First, let us compute the first derivative of $\mathcal{F}$. We skip the details of a very laborious calculation to give

$$
\begin{aligned}
\mathcal{F}^{\prime}(\alpha)= & \frac{\partial^{2} \varphi}{\partial \alpha^{2}}(\Sigma) \frac{\partial \phi}{\partial \alpha}\left(\bar{\Sigma}^{e q}\right)-\frac{\partial^{2} \phi}{\partial \alpha^{2}}(\Sigma) \frac{\partial \varphi}{\partial \alpha}\left(\bar{\Sigma}^{e q}\right) \\
& +\left(\frac{\partial \varphi}{\partial \alpha}(\Sigma) \nabla_{\Sigma}\left(\frac{\partial \phi}{\partial \alpha}\right)\left(\bar{\Sigma}^{e q}\right)-\frac{\partial \phi}{\partial \alpha}(\Sigma) \nabla_{\Sigma}\left(\frac{\partial \varphi}{\partial \alpha}\right)\left(\bar{\Sigma}^{e q}\right)\right) \cdot V,
\end{aligned}
$$

where $V=\left(\tilde{\tau}_{\alpha}, \tilde{e}_{\alpha}, \tilde{\pi}_{\alpha}\right)^{T}$ with

$$
\begin{aligned}
& \tilde{\tau}_{\alpha}=\frac{1}{D(\bar{\sigma})}\left(\frac{\partial \phi}{\partial e}\left(\bar{\Sigma}^{e q}\right) \frac{\partial \varphi}{\partial \alpha}(\Sigma)-\frac{\partial \varphi}{\partial e}\left(\bar{\Sigma}^{e q}\right) \frac{\partial \phi}{\partial \alpha}(\Sigma)+\frac{\partial \pi}{\partial e}(\bar{\sigma}) \mathcal{F}(\alpha)\right), \\
& \tilde{e}_{\alpha}=\frac{1}{D(\bar{\sigma})}\left(\frac{\partial \varphi}{\partial \tau}\left(\bar{\Sigma}^{e q}\right) \frac{\partial \phi}{\partial \alpha}(\Sigma)-\frac{\partial \phi}{\partial \tau}\left(\bar{\Sigma}^{e q}\right) \frac{\partial \varphi}{\partial \alpha}(\Sigma)-\frac{\partial \pi}{\partial \tau}(\bar{\sigma}) \mathcal{F}(\alpha)\right), \\
& \tilde{\pi}_{\alpha}=\tilde{\tau}_{\alpha} \frac{\partial \pi}{\partial \tau}(\bar{\sigma})+\tilde{e}_{\alpha} \frac{\partial \pi}{\partial e}(\bar{\sigma}) .
\end{aligned}
$$


Now, we evaluate this derivative at point $\alpha_{0}$, a root of $\mathcal{F}$. To simplify the notation, we set $\Sigma_{0}=\left(\tau, e, \alpha_{0}\right)^{T}$ and

$$
\bar{\Sigma}_{0}^{e q}=\left(\bar{\tau}\left(\varphi\left(\Sigma_{0}\right), \phi\left(\Sigma_{0}\right)\right), \bar{e}\left(\varphi\left(\Sigma_{0}\right), \phi\left(\Sigma_{0}\right)\right), \bar{\pi}\left(\bar{\tau}\left(\varphi\left(\Sigma_{0}\right), \phi\left(\Sigma_{0}\right)\right), \bar{e}\left(\varphi\left(\Sigma_{0}\right), \phi\left(\Sigma_{0}\right)\right)\right)\right) .
$$

By definition, we have $\mathcal{F}\left(\alpha_{0}\right)=0$. In addition, since $\alpha \mapsto \varphi(\alpha)$ is monoton, we can introduce the function $\left(\Sigma_{1}, \Sigma_{2}\right) \mapsto \gamma\left(\Sigma_{1}, \Sigma_{2}\right)$ defined by (3.9) to deduce from $\mathcal{F}\left(\alpha_{0}\right)=0$ the following relation:

$$
\frac{\partial \phi}{\partial \alpha}\left(\Sigma_{0}\right)=\gamma\left(\bar{\Sigma}_{0}^{e q}, \Sigma_{0}\right) \frac{\partial \phi}{\partial \alpha}\left(\bar{\Sigma}_{0}^{e q}\right)
$$

By plugging this identity into $\mathcal{F}^{\prime}$, we get

$$
\mathcal{F}^{\prime}\left(\alpha_{0}\right)=\mathcal{H}\left(\bar{\Sigma}_{0}^{e q}, \Sigma_{0}\right)
$$

where $\mathcal{H}$ is defined by (3.8). Since for all $\left(\Sigma_{1}, \Sigma_{2}\right)$ we have $\mathcal{H}\left(\Sigma_{1}, \Sigma_{2}\right) \neq 0$ then $\mathcal{H}$ never changes of sign and thus $\mathcal{F}^{\prime}\left(\alpha_{0}\right)$ has the same sign for all roots of $\mathcal{F}$. The proof is achieved.

4. Applications. The purpose of this section is to apply our main result by revisiting several well-known schemes: Godunov scheme, Osher scheme, and HLLC scheme. Next, we derive a new family of entropy preserving schemes.

4.1. The Godunov scheme. In the framework of the Godunov scheme, the intermediate state $U^{\star}\left(\frac{x}{t} ; U_{L}, U_{R}\right)$ involved in (2.1) is made of the exact Riemann solution which is given by the following four constant states:

$U_{L}$ and $U_{L}^{\star}$ connected by a 1 -shock or a 1 -rarefaction,

$U_{L}^{\star}$ and $U_{R}^{\star}$ connected by a 2 -contact discontinuity,

$U_{R}^{\star}$ and $U_{R}$ connected by a 3 -shock or a 3 -rarefaction.

To apply our main results Theorem 3.1 and Theorem 3.3 , we first exhibit the additional half consistency condition (3.2). To address such an issue, we fix $a^{\star}=u^{\star}$ the exact contact discontinuity velocity involved by the exact Riemann solution, and we consider the Riemann solution made of the three constant states: $U_{L}^{\star}, U_{R}^{\star}$ and $U_{R}$ so that we have

$$
U^{\star}\left(\frac{x}{t} ; U_{L}^{\star}, U_{R}\right)=\left\{\begin{array}{l}
U_{L}^{\star} \quad \text { if } \frac{x}{t}<u^{\star}, \\
U^{\star}\left(\frac{x}{t} ; U_{L}, U_{R}\right) \quad \text { if } \frac{x}{t}>u^{\star} .
\end{array}\right.
$$

By integrating this Riemann solution, we obtain

$$
\begin{aligned}
\frac{1}{\Delta x} \int_{-\frac{\Delta x}{2}}^{\frac{\Delta x}{2}} \rho_{\mathcal{R}}\left(\frac{x}{\Delta t} ; U_{L}^{\star}, U_{R}\right) d x \\
\quad=\frac{1}{\Delta x} \int_{-\frac{\Delta x}{2}}^{u^{\star} \Delta t} \rho_{\mathcal{R}}\left(\frac{x}{\Delta t} ; U_{L}^{\star}, U_{R}\right) d x+\frac{1}{\Delta x} \int_{u^{\star} \Delta t}^{\frac{\Delta x}{2}} \rho_{\mathcal{R}}\left(\frac{x}{\Delta t} ; U_{L}, U_{R}\right) d x \\
=\frac{1}{2} \rho_{L}^{\star}+\frac{\Delta t}{\Delta x} \rho_{L}^{\star} u_{L}^{\star}+\frac{1}{\Delta x} \int_{u^{\star} \Delta t}^{\frac{\Delta x}{2}} \rho_{\mathcal{R}}\left(\frac{x}{\Delta t} ; U_{L}, U_{R}\right) d x
\end{aligned}
$$

But, we also have

$$
\frac{1}{\Delta x} \int_{-\frac{\Delta x}{2}}^{\frac{\Delta x}{2}} \rho_{\mathcal{R}}\left(\frac{x}{\Delta t} ; U_{L}^{\star}, U_{R}\right) d x=\frac{1}{2}\left(\rho_{L}^{\star}+\rho_{R}\right)-\frac{\Delta t}{\Delta x}\left(\rho_{R} u_{R}-\rho_{L}^{\star} u_{L}^{\star}\right) .
$$


From (4.1) and (4.2) we immediately deduce the expected relation (3.2):

$$
\frac{1}{\Delta x} \int_{u^{\star} \Delta t}^{\frac{\Delta x}{2}} \rho_{\mathcal{R}}\left(\frac{x}{\Delta t} ; U_{L}^{\star}, U_{R}\right) d x=\frac{1}{2} \rho_{R}-\frac{\Delta t}{\Delta x} \rho_{R} u_{R} .
$$

Now, we independently consider each half Riemann solution respectively given for $\frac{x}{t}<u^{\star}$ and $\frac{x}{t}>u^{\star}$. We just study for the right half Riemann solution (the same arguments can be applied to the left). Two cases must be consider according to the connection between $U_{R}^{\star}$ and $U_{R}$.

If $U_{R}^{\star}$ and $U_{R}$ are connected by a 3-rarefaction, by definition of the Euler rarefaction (see $[18,25])$ the specific entropy stays invariant and we directly have:

$$
s\left(\tau_{R}^{\star}\left(\frac{x}{t} ; U_{L}, U_{R}\right), e_{R}^{\star}\left(\frac{x}{t} ; U_{L}, U_{R}\right)\right)=s_{R}, \quad \frac{x}{t}>u^{\star} .
$$

Next, if $U_{R}^{\star}$ and $U_{R}$ are connected by a 3 -shock, we suggest to apply Theorem 3.3 to establish (3.3b). Let us recall that a 3 -shock with speed $\delta$ is characterized by the following Rankine-Hugoniot relations:

$$
\left\{\begin{array}{l}
-\delta\left(\rho_{R}-\rho_{R}^{\star}\right)+\left(\rho_{R} u_{R}-\rho_{R}^{\star} u_{R}^{\star}\right)=0, \\
-\delta\left(\rho_{R} u_{R}-\rho_{R}^{\star} u_{R}^{\star}\right)+\left(\rho_{R}\left(u_{R}\right)^{2}+p_{R}-\rho_{R}^{\star}\left(u_{R}^{\star}\right)^{2}-p_{R}^{\star}\right)=0, \\
-\delta\left(\rho_{R} E_{R}-\rho_{R}^{\star} E_{R}^{\star}\right)+\left(\left(\rho_{R} E_{R}+p_{R}\right) u_{R}-\left(\rho_{R}^{\star} E_{R}^{\star}+p_{R}^{\star}\right) u_{R}^{\star}\right)=0 .
\end{array}\right.
$$

It suffices to fix

$$
\varphi(\tau, e, \alpha)=\alpha+m^{2} \tau \quad \text { and } \quad \phi(\tau, e, \alpha)=e-\frac{\alpha^{2}}{2 m^{2}},
$$

where $\mathrm{m}$ is the invariant momentum in the schock frame $m=\rho(u-\sigma)$ to obtain

$$
\varphi\left(\tau_{R}, e_{R}, p\left(\tau_{R}, e_{R}\right)\right)=\varphi\left(\tau_{R}^{\star}, e_{R}^{\star}, p_{R}^{\star}\right) \quad \text { and } \quad \phi\left(\tau_{R}, e_{R}, p\left(\tau_{R}, e_{R}\right)\right)=\phi\left(\tau_{R}^{\star}, e_{R}^{\star}, p_{R}^{\star}\right) .
$$

As a consequence, we can apply Theorem 3.3 as soon as the triple $(\varphi, \phi, p)$ satisfies the assumptions (i)-(ii)-(iii) Proposition 3.2. Here we have:

$$
\begin{aligned}
& D(\tau, e)=m^{2}-\left(p(\tau, e) \frac{\partial p}{\partial e}(\tau, e)-\frac{\partial p}{\partial \tau}(\tau, e)\right), \\
& J(\tau, e, \alpha)=p(\tau, e)-\alpha, \\
& K(\tau, e, \alpha)=-1 \\
& \mathcal{H}\left(\left(\tau_{1}, e_{1}, \alpha_{1}\right),\left(\tau_{2}, e_{2}, \alpha_{2}\right)\right)=\frac{1}{D\left(\tau_{1}, e_{1}\right)}
\end{aligned}
$$

and all the assumptions are obviously satisfied.

From the above Godunov scheme presentation, the main idea to derive approximate Riemann solver stays in the preservation of the exact rarefaction waves (Osher scheme) or shock waves (HLLC scheme). Arguing the above comments, we have just saw that exact rarefaction or shock waves yield to the expected entropy minimum principle and the resulting scheme will be entropy preserving. In the following section we give more details about the HLLC scheme. 
4.2. Two constant intermediate state solvers. We adopt an approximate Riemann solver made of two constant intermediate states. The resulting approximate Riemann solver reads:

$$
U_{\mathcal{R}}^{\Delta x}\left(\frac{x}{t} ; U_{L}, U_{R}\right)= \begin{cases}U_{L}, & \text { if } \frac{x}{t}<a^{-}, \\ U_{L}^{\star}, & \text { if } a^{-}<\frac{x}{t}<a^{\star}, \\ U_{R}^{\star}, & \text { if } a^{\star}<\frac{x}{t}<a^{+}, \\ U_{R}, & \text { if } \frac{x}{t}>a^{+} .\end{cases}
$$

By arguing the consistency conditions (2.4) and (3.2), from the density we easily obtain:

$$
\rho_{L}^{\star}=\frac{a^{-}-u_{L}}{a^{-}-a^{\star}} \rho_{L} \quad \text { and } \quad \rho_{R}^{\star}=\frac{a^{+}-u_{R}}{a^{+}-a^{\star}} \rho_{R} .
$$

To simplify the notations, let us set

$$
a^{-}=u_{L}-b / \rho_{L} \quad \text { and } \quad a^{+}=u_{R}+b / \rho_{R} \quad \text { with } b>0 .
$$

Next, by considering the consistency condition (2.4) applied to the momentum and the total energy, a straightforward computation gives:

$$
\left\{\begin{array}{l}
u_{L}^{\star}+u_{R}^{\star}=u_{L}+u_{R}-\frac{1}{b}\left(p_{R}-p_{L}\right), \\
\frac{1}{2}\left(\left(u_{L}^{\star}\right)^{2}+\left(u_{R}^{\star}\right)^{2}\right)=\left(E_{L}-e_{L}^{\star}\right)+\left(E_{R}-e_{R}^{\star}\right)-\frac{1}{b}\left(p_{R} u_{R}-p_{L} u_{L}\right),
\end{array}\right.
$$

where $e_{L, R}^{\star}=E_{L, R}^{\star}-\frac{\left(u_{L, R}^{\star}\right)^{2}}{2}$. To solve this system, we have to determine suitable values for $e_{L}^{\star}$ and $e_{R}^{\star}$. To enforce the required entropy minimum principle, we adopt the invariance principle (3.12)-(3.15) for some triples $\left(\varphi^{L}, \phi^{L}, \pi^{L}\right)$ and $\left(\varphi^{R}, \phi^{R}, \pi^{R}\right)$, to get

$$
\left\{\begin{array}{l}
\varphi\left(\tau_{L}, e_{L}, \pi^{L}\left(\tau_{L}, e_{L}\right)\right)=\varphi\left(\tau_{L}^{\star}, e_{L}^{\star}, \alpha_{L}^{\star}\right) \\
\phi\left(\tau_{L}, e_{L}, \pi^{L}\left(\tau_{L}, e_{L}\right)\right)=\phi\left(\tau_{L}^{\star}, e_{L}^{\star}, \alpha_{L}^{\star}\right)
\end{array}\right.
$$

and

$$
\left\{\begin{array}{l}
\varphi\left(\tau_{R}, e_{R}, \pi^{R}\left(\tau_{R}, e_{R}\right)\right)=\varphi\left(\tau_{R}^{\star}, e_{R}^{\star}, \alpha_{R}^{\star}\right), \\
\phi\left(\tau_{R}, e_{R}, \pi^{R}\left(\tau_{R}, e_{R}\right)\right)=\phi\left(\tau_{R}^{\star}, e_{R}^{\star}, \alpha_{R}^{\star}\right) .
\end{array}\right.
$$

Both systems (4.4) and (4.5), if solvable, can give $\left(E_{L}^{\star}, \alpha_{L}^{\star}\right)$ and $\left(E_{R}^{\star}, \alpha_{R}^{\star}\right)$.

For instance and according to the triple $(\varphi, \phi, \pi)$ obtained for the Godunov scheme, we propose to consider the following choice:

$$
\left\{\begin{array}{l}
\varphi^{L, R}(\tau, e, \alpha)=\alpha+m^{2} \tau, \\
\phi^{L, R}(\tau, e, \alpha)=e-\frac{\alpha^{2}}{2 m^{2}},
\end{array} \quad \text { and } \quad \pi^{L, R}(\tau, e)=p(\tau, e),\right.
$$

which satisfies all the required assumptions as soon as the constant $m>0$ is large enough. 
By solving (4.4) and (4.5) we obtain:

$$
\begin{aligned}
& e_{L}^{\star}=e_{L}+\frac{a^{\star}-u_{L}}{b}\left(\frac{m^{2}}{2} \frac{a^{\star}-u_{L}}{b}-p_{L}\right), \\
& e_{R}^{\star}=e_{R}+\frac{a^{\star}-u_{R}}{b}\left(\frac{m^{2}}{2} \frac{a^{\star}-u_{R}}{b}+p_{R}\right) .
\end{aligned}
$$

These relations are plug into (4.3) to determine $u_{L}^{\star}$ and $u_{R}^{\star}$. We now have to fix $a^{\star}$ and $b$ such that $\rho_{L, R}^{\star}>0, e_{L, R}^{\star}>0$ and $u_{L}-\frac{b}{\rho_{L}}<a^{\star}<u_{R}+\frac{b}{\rho_{R}}$. For instance, we suggest to fix $a^{\star}$ as follows:

$$
a^{\star}=\frac{1}{2}\left(u_{L}+u_{R}\right)-\frac{b}{2 m^{2}}\left(p_{R}-p_{L}\right) \pm \frac{1}{2 m^{2}} \sqrt{\left(b^{2}-m^{2}\right)\left(m^{2}\left(u_{L}-u_{R}\right)^{2}+\left(p_{R}-p_{L}\right)^{2}\right)},
$$

with $b \geq m$. The solution of system (4.3) is thus given by

$$
u_{L}^{\star}=u_{R}^{\star}=\frac{1}{2}\left(u_{L}+u_{R}\right)-\frac{1}{2 b}\left(p_{R}-p_{L}\right) .
$$

We note that the HLLC scheme (or equivalently the Suliciu relaxation scheme) coincides with the choice $b=m$, but the general formula (4.7) also gives an entropy preserving approximate Riemann solver family.

To conclude this section, let us note that we have restricted the present scheme derivation for a triple $(\varphi, \phi, \pi)$ which coincides with an exact shock invariance, and the intermediate states have been assumed to be constant. The reader will easily be convinced for possible extensions involving other triple choice $(\varphi, \phi, \pi)$ but also non constant intermediate states as shown in the next section.

4.3. Two affine intermediate state solvers. We now consider an approximate Riemann solver made of two affine intermediate states. The corresponding approximate solver writes:

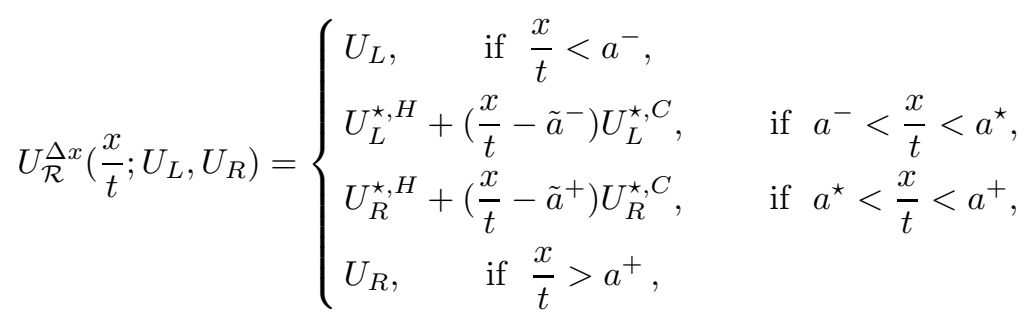

where $U_{L}^{\star, H}, U_{R}^{\star, H}, U_{L}^{\star, C}$ and $U_{R}^{\star, C}$ are constant states:

$$
\begin{gathered}
U_{L}^{\star, H}=\left(\begin{array}{c}
\rho_{L}^{\star, H} \\
\rho_{L}^{\star, H} u_{L}^{\star, H} \\
\rho_{L}^{\star, H} E_{L}^{\star, H}
\end{array}\right), U_{R}^{\star, H}=\left(\begin{array}{c}
\rho_{R}^{\star, H} \\
\rho_{R}^{\star, H} u_{R}^{\star, H} \\
\rho_{R}^{\star, H} E_{R}^{\star, H}
\end{array}\right), \\
U_{L}^{\star, C}=\left(\begin{array}{c}
\beta_{L}^{\star, C} \\
\gamma_{L}^{\star, C} \\
\delta_{L}^{\star, C}
\end{array}\right), U_{R}^{\star, C}=\left(\begin{array}{c}
\beta_{R}^{\star, C} \\
\gamma_{R}^{\star, C} \\
\delta_{R}^{\star, C}
\end{array}\right) .
\end{gathered}
$$

The speeds $\tilde{a}^{-}$and $\tilde{a}^{+}$are given by:

$$
\tilde{a}^{-}=\frac{a^{-}+a^{\star}}{2}, \quad \tilde{a}^{+}=\frac{a^{\star}+a^{+}}{2} .
$$


Such affine scheme has been introduced in the case of one intermediate state and known as HLLEM and HLLEMR solvers [11]. The two intermediate version considered here seems to be new up to our knowledge.

This approximate solver is fully determined as soon as $U_{L}^{\star, H}, U_{R}^{\star, H}, U_{L}^{\star, C}$ and $U_{R}^{\star, C}$ are characterized. The consistency conditions (2.4) and (3.2) lead to:

$$
\rho_{L}^{\star, H}=\frac{a^{-}-u_{L}}{a^{-}-a^{\star}} \rho_{L} \quad \text { and } \quad \rho_{R}^{\star, H}=\frac{a^{+}-u_{R}}{a^{+}-a^{\star}} \rho_{R} .
$$

By applying the consistency condition (2.4) to the momentum and the total energy, one gets:

$$
\left\{\begin{array}{l}
u_{L}^{\star, H}+u_{R}^{\star, H}=u_{L}+u_{R}-\frac{1}{b}\left(p_{R}-p_{L}\right), \\
E_{L}-E_{L}^{\star, H}+E_{R}-E_{R}^{\star, H}=\frac{p_{R} u_{R}-p_{L} u_{L}}{b},
\end{array}\right.
$$

where

$$
a^{-}=u_{L}-b / \rho_{L} \quad \text { and } \quad a^{+}=u_{R}+b / \rho_{R} \quad \text { with } b>0
$$

At this level of the derivation of the solver (4.8), we notice that equations (4.9), (4.10) are not sufficient to characterize all the components of $U_{L}^{\star}, H, U_{R}^{\star, H}, U_{L}^{\star}, C$ and $U_{R}^{\star, C}$. We then use the entropy maximum principle with invariance principle (3.12)-(3.15) as in (4.4)-(4.5) with the following choice

$$
\left\{\begin{array}{l}
\varphi^{L, R}(\tau, e, \alpha)=-\alpha+m^{2} \tau, \\
\phi^{L, R}(\tau, e, \alpha)=e-\frac{\alpha^{2}}{2 m^{2}},
\end{array} \quad \text { and } \quad \pi^{L, R}(\tau, e)=p(\tau, e) .\right.
$$

The triple $(\varphi, \phi, p)$ satisfies the assumptions (i)-(ii)-(iii) Proposition 3.2. Indeed, we have:

$$
\begin{aligned}
& D(\tau, e)=m^{2}-\left(p(\tau, e) \frac{\partial p}{\partial e}(\tau, e)+\frac{\partial p}{\partial \tau}(\tau, e)\right) \\
& J(\tau, e, \alpha)=-p(\tau, e)-\alpha \\
& K(\tau, e, \alpha)=1 \\
& \mathcal{H}\left(\left(\tau_{1}, e_{1}, \alpha_{1}\right),\left(\tau_{2}, e_{2}, \alpha_{2}\right)\right)=-\frac{1}{D\left(\tau_{1}, e_{1}\right)} .
\end{aligned}
$$

The equilibrium is reached at $\alpha=-p(\tau, e)$ and $D(\tau, e)>0$ as soon as $m$ is large enough. We get also

$$
\left\{\begin{array}{l}
e_{L}^{\star}=e_{L}+\left(p_{L}-\frac{m^{2}}{2}\left(\tau_{L}-\tau_{L}^{\star}\right)\right)\left(\tau_{L}-\tau_{L}^{\star}\right) \\
e_{R}^{\star}=e_{R}+\left(p_{R}-\frac{m^{2}}{2}\left(\tau_{R}-\tau_{R}^{\star}\right)\right)\left(\tau_{R}-\tau_{R}^{\star}\right)
\end{array}\right.
$$

Here, $\tau_{L, R}^{\star}, e_{L, R}^{\star}$ are respectively specific volumes and internal energies associated to 
$U_{L, R}^{\star, H}+\left(\frac{x}{\Delta t}-\tilde{a}^{-,+}\right) U_{L, R}^{\star, C}$ given by:

$$
\left\{\begin{array}{l}
\tau_{L}^{\star}=\frac{1}{\rho_{L}^{\star, H}+\left(\frac{x}{\Delta t}-\tilde{a}^{-}\right) \beta_{L}^{\star, C}}, \\
\tau_{R}^{\star}=\frac{1}{\rho_{R}^{\star, H}+\left(\frac{x}{\Delta t}-\tilde{a}^{+}\right) \beta_{R}^{\star, C}}, \\
e_{L}^{\star}=\frac{\rho_{L}^{\star, H} E_{L}^{\star, H}+\left(\frac{x}{\Delta t}-\tilde{a}^{-}\right) \delta_{L}^{\star, C}-\frac{1}{2} \frac{\left(\rho_{L}^{\star, H} u_{L}^{\star, H}+\left(\frac{x}{\Delta t}-\tilde{a}^{-}\right) \gamma_{L}^{\star}, C\right.}{\rho_{L}^{\star, H}+\left(\frac{x}{\Delta t}-\tilde{a}^{-}\right) \beta_{L}^{\star, C}}}{\rho_{L}^{\star, H}+\left(\frac{x}{\Delta t}-\tilde{a}^{-}\right) \beta_{L}^{\star, C}} \\
e_{R}^{\star}=\frac{\rho_{R}^{\star, H} E_{R}^{\star, H}+\left(\frac{x}{\Delta t}-\tilde{a}^{+}\right) \delta_{R}^{\star, C}-\frac{1}{2} \frac{\left(\rho_{R}^{\star, H} u_{R}^{\star, H}+\left(\frac{x}{\Delta t}-\tilde{a}^{+}\right) \gamma_{R}^{\star, C}\right)^{2}}{\rho_{R}^{\star, H}+\left(\frac{x}{\Delta t}-\tilde{a}^{+}\right) \beta_{R}^{\star, C}}}{\rho_{R}^{\star, H}+\left(\frac{x}{\Delta t}-\tilde{a}^{+}\right) \beta_{R}^{\star, C}}
\end{array}\right.
$$

According to (4.12), internal energies $e_{L, R}^{\star}$ are positive as soon as

$$
|m|>\frac{1}{\sqrt{2}} \max \left(\frac{p_{L}}{e_{L}}, \frac{p_{R}}{e_{R}}\right) .
$$

Moreover, by taking $a^{-}<u_{L}$ and $a^{\star}>a^{-}$we get $\rho_{L}^{\star}>0$, and by chosing $a^{+}>u_{R}$ and $a^{\star}<a^{+}$we obtain $\rho_{R}^{\star}>0$. The system still remains undetermined, especially the constant states $U_{L}^{\star, C}$ and $U_{R}^{\star, C}$ are not fully characterized. We then suggest to limit $\beta_{L, R}^{\star, C}$ in order to maintain

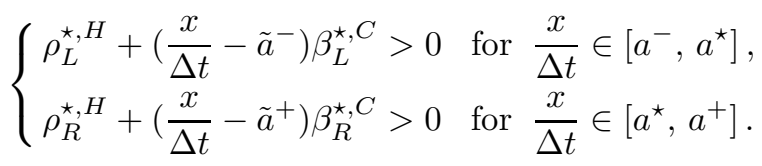

By using the monotonicity of the functions $\frac{x}{\Delta t} \mapsto \rho_{L, R}^{\star, H}+\left(\frac{x}{\Delta t}-\tilde{a}^{-,+}\right) \beta_{L, R}^{\star, C}$ and then evaluating them on the endpoints $a^{\star}, a^{+,-}$, conditions (4.14) are equivalent to

$$
\left\{\begin{array}{l}
\left|\beta_{L}^{\star, C}\right|<\frac{2\left(u_{L}-a^{-}\right)}{\left(a^{\star}-a^{-}\right)^{2}} \rho_{L}, \\
\left|\beta_{R}^{\star, C}\right|<\frac{2\left(a^{+}-u_{R}\right)}{\left(a^{+}-a^{\star}\right)^{2}} \rho_{R} .
\end{array}\right.
$$

In order to get the missing constants $\gamma_{L}^{\star, C}, \gamma_{R}^{\star, C}, \delta_{L}^{\star, C}$ and $\delta_{R}^{\star, C}$, we proceed as follows. By chosing

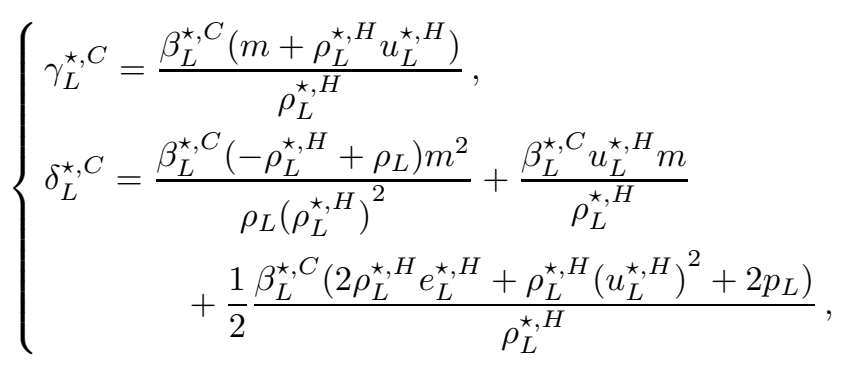

expressions of $e_{L}^{\star}$ withdrawn from equations (4.12) and (4.13) are identical. The 
following choice

$$
\left\{\begin{aligned}
\gamma_{R}^{\star, C}= & \frac{\beta_{R}^{\star, C}\left(m+\rho_{R}^{\star, H} u_{R}^{\star, H}\right)}{\rho_{R}^{\star, H}}, \\
\delta_{R}^{\star, C}= & \frac{\beta_{R}^{\star, C}\left(-\rho_{R}^{\star, H}+\rho_{R}\right) m^{2}}{\rho_{R}\left(\rho_{R}^{\star, H}\right)^{2}}+\frac{\beta_{R}^{\star, C} u_{R}^{\star, H} m}{\rho_{R}^{\star, H}} \\
& +\frac{1}{2} \frac{\beta_{R}^{\star, C}\left(2 \rho_{L}^{\star, H} e_{R}^{\star, H}+\rho_{R}^{\star, H}\left(u_{R}^{\star, H}\right)^{2}+2 p_{R}\right)}{\rho_{R}^{\star, H}},
\end{aligned}\right.
$$

makes equal expressions of $e_{R}^{\star}$ taken from equations (4.12) and (4.13).

The identity

$$
u_{L}^{\star, H}=u_{R}^{\star, H}=\frac{1}{2}\left(u_{L}+u_{R}\right)-\frac{1}{2 b}\left(p_{R}-p_{L}\right),
$$

solves the first equation of system (4.10). The star speed $a^{\star}$ given by

$$
\begin{aligned}
a^{\star}=\frac{1}{2}\left(u_{L}\right. & \left.+u_{R}\right)-\frac{b}{2 m^{2}}\left(p_{R}-p_{L}\right) \\
& \pm \frac{1}{2 m^{2}} \sqrt{\left(b^{2}+m^{2}\right)\left[\left(p_{R}-p_{L}\right)+m\left(u_{L}-u_{R}\right)\right]\left[\left(p_{R}-p_{L}\right)-m\left(u_{L}-u_{R}\right)\right]},
\end{aligned}
$$

solves the second equation of system (4.10). Then all the components of $U_{L}^{\star, H}, U_{R}^{\star, H}$, $U_{L}^{\star, C}$ and $U_{R}^{\star, C}$ are determined. We have thus derived a new two affine intermediate states entropic solver.

5. Conclusion. In the present work, we have proposed a generalization of the entropy preserving property for several schemes. More specifically, this work extends the proof of discrete entropy inequalities satisfied by the HLLC scheme (see [7]). Moreover, the suggested extension just considers the associated approximate Riemann solver and no additional ad-hoc model (kinetic models or relaxation models) are involved. This approach turns out to be a little more restrictive than the well-known Harten-Lax-van Leer Theorem, but with the benefit to be easily applied. In addition, the adopted approach yields to derivations of full families of entropy preserving approximate Riemann solvers.

The derivation here has been performed in the framework of the general Euler equations. Of course, this can be extended to several hyperbolic systems of conservation laws with the same structure as Saint-Venant model or 10-moment model (for instance). Since our approach does not impose an approximate Riemann solver made of constant states, we can also plan to use this work to consider high-order approximate Riemann solvers.

\section{REFERENCES}

[1] C. Berthon. Inégalités d'entropie pour un schéma de relaxation. C. R. Math. Acad. Sci. Paris, 340(1):63-68, 2005.

[2] C. Berthon. Stability of the MUSCL schemes for the Euler equations. Commun. Math. Sci., 3(2):133-157, 2005.

[3] C. Berthon. Numerical approximations of the 10-moment Gaussian closure. Math. Comp., 75(256):1809-1831 (electronic), 2006.

[4] C. Berthon, P. Charrier and B. Dubroca, An HLLC scheme to solve the M1 model of radiative transfer in two space dimensions, J. Sci. Comput., 31(3):347-389, 2007. 
[5] C. Berthon and F. Marche. A positive preserving high order VFRoe scheme for shallow water equations: a class of relaxation schemes. SIAM J. Sci. Comput., 30(5):2587-2612, 2008.

[6] F. Bouchut. Entropy satisfying flux vector splittings and kinetic BGK models. Numer. Math., 94(4):623-672, 2003.

[7] F. Bouchut. Nonlinear stability of finite volume methods for hyperbolic conservation laws and well-balanced schemes for sources. Frontiers in Mathematics. Birkhäuser Verlag, Basel, 2004.

[8] M.O. Bristeau and B. Perthame. Kinetic schemes for solving Saint-Venant equations on unstructured grids. In Innovative methods for numerical solutions of partial differential equations (Arcachon, 1998), pages 267-277. World Sci. Publ., River Edge, NJ, 2002.

[9] T. Buffard, T. Gallouët, and J.-M. Hérard. A sequel to a rough Godunov scheme: application to real gases. Comput. \& Fluids, 29(7):813-847, 2000.

[10] C. Chalons. Bilans d'entropie discrets dans l'approximation numérique des chocs non classiques. Application aux équations de NS multi-pression $2 D$ et à quelques systèmes viscocapillaires. PhD thesis, Ecole Polytechnique, 2002.

[11] P. Charrier, B. Dubroca, L. Flandrin, Un solveur de Riemann approché pour 1'étude d'écoulements hypersoniques bidimensionnels, C. R. Math. Acad. Sci. Paris, 317, 10831086, 1993.

[12] F. Coquel and B. Perthame. Relaxation of energy and approximate Riemann solvers for general pressure laws in fluid dynamics. SIAM J. Numer. Anal., 35(6):2223-2249 (electronic), 1998.

[13] B. Engquist and S. Osher. Stable and entropy satisfying approximations for transonic flow calculations, Math. Comput., 34:45-75, 1980.

[14] G. Gallice, Solveurs simples positifs et entropiques pour les systèmes hyperboliques avec terme source. (French) [Entropic Godunov-type schemes for hyperbolic systems with source term], C. R. Math. Acad. Sci. Paris 334(8):713-716, 2002.

[15] G. Gallice, Schémas positifs et solveurs de Riemann simples entropiques pour des systèmes hyperboliques avec terme source: application à la dynamique des gaz avec gravité. Prepint MAB-LRC 02.05, Université Bordeaux 1, France.

[16] G. Gallice, Positive and Entropy Stable Godunov-type Schemes for Gas Dynamics and MHD Equations in Lagrangian or Eulerian Coordinates, Numer. Math., 94(4):673-713, 2003.

[17] T. Gallouët, J.-M. Hérard, and N. Seguin. On the use of symmetrizing variables for vacuums. Calcolo, 40(3):163-194, 2003.

[18] E. Godlewski and P.A. Raviart. Numerical approximation of hyperbolic systems of conservation laws, volume 118 of Applied Mathematical Sciences. Springer-Verlag, New York, 1996.

[19] A. Harten and P. D. Lax. A random choice finite difference scheme for hyperbolic conservation laws. SIAM J. Numer. Anal., 18(2):289-315, 1981.

[20] A. Harten, P. D. Lax, C. D. Levermore, and W. J. Morokoff. Convex entropies and hyperbolicity for general Euler equations. SIAM J. Numer. Anal., 35(6):2117-2127 (electronic), 1998.

[21] A. Harten, P.D. Lax, and B. Van Leer. On upstream differencing and Godunov-type schemes for hyperbolic conservation laws. SIAM Rev., 25(1):35-61, 1983.

[22] S. Jin and Z. P. Xin. The relaxation schemes for systems of conservation laws in arbitrary space dimensions. Comm. Pure Appl. Math., 48(3):235-276, 1995.

[23] B. Khobalatte and B. Perthame. Maximum principle on the entropy and second-order kinetic schemes. Math. Comp., 62(205):119-131, 1994.

[24] B. Van Leer. Towards the ultimate conservative difference scheme v. a second order sequel to godunov's method. J. Comput. Phys., 32:101-136, 1979.

[25] Randall J. LeVeque. Finite volume methods for hyperbolic problems. Cambridge Texts in Applied Mathematics. Cambridge University Press, Cambridge, 2002.

[26] J.-M. Masella, I. Faille, and T. Gallouët. On an approximate Godunov scheme. Int. J. Comput. Fluid Dyn., 12(2):133-149, 1999.

[27] P. L. Roe. Approximate Riemann solvers, parameter vectors, and difference schemes. J. Comput. Phys., 43(2):357-372, 1981.

[28] A. Sangam, An HLLC scheme for ten-moments approximation coupled with magnetic field, Int. J. Comput. Sci. Math., 2(1-2):73-109, 2008.

[29] D. Serre. Systems of conservation laws. 1. Cambridge University Press, Cambridge, 1999. Hyperbolicity, entropies, shock waves, Translated from the 1996 French original by I. N. Sneddon.

[30] E. Tadmor, A minimum entropy principle in the gas dynamics equations. Appl. Numer. Math., 2(3-5):211-219, 1986

[31] E. F. Toro, M. Spruce, and W. Speare. Restoration of the contact surface in the hll riemann solver. Shock waves, 4:25-34, 1994.

[32] E.F. Toro, Riemann solvers and numerical methods for fluid dynamics. Springer-Verlag, Berlin, 
third edition, 2009. A practical introduction.

[33] Y. Xing and C.W. Shu. High-order well-balanced finite difference weno schemes for a class of hyperbolic systems with source terms. Journal of Scientific Computing, 27(1-3):477-494, 2006. 